\title{
ADAMANTIOS CORAY ET SES CORRECTIONS INÉDITES SUR LUCIEN: LE MS. CHIOS 490, P. 455-576 ${ }^{1}$
}

\author{
Orestis Karavas* \\ Université du Péloponnèse
}

RESUMO: No presente artigo, apresentam-se 254 das quase 1200 correções de Adamantios Coray sobre o texto de Luciano, as quais, contudo, continuam inéditas e escritas à mão em três lugares diferentes: nos manuscritos 379 e 490 de Quios e às margens da editio Bipontina, que o mesmo Coary consultava. Escolhemos as correções que não foram propostas por nenhum filólogo até o presente momento. Muitas delas iluminam algumas passagens obscuras e incompreensíveis do corpus luciânico, e põem em evidência os profundos conhecimentos da língua e da literatura gregas por Coray, assim como sua surpreendente intuição linguística.

PALAVRAS-CHAVE: Adamantios Coray; Luciano; manuscritos de Quios 379 e 490; correções textuais.

\section{À la mémoire de Jacques Bompaire}

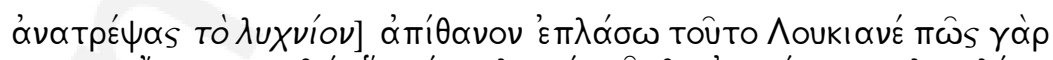

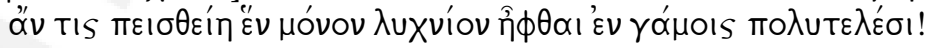

Note marginale de Coray sur Luc., Symp. 46

\footnotetext{
^okaravas@hotmail.com

${ }^{1}$ Une lecture d'une version incomplète du présent article a été prononcée dans le cadre du colloque Tpoфeî à Rethymnon (23-25/5/2008). Je voudrais remercier les professeurs J. L. Brandão, G. A. Christodoulou, H. G. Nesselrath et mon amie Isabelle Gassino pour l'aide précieuse qu'ils m'ont apportée dans la réalisation de ce travail.
} 
Q damantios Coray, médecin et philologue grec, est né à Smyrne en 1748 et mort à Paris en 1833. Il est très connu pour ses thèses concernant la langue grecque moderne et plus exactement pour la voie

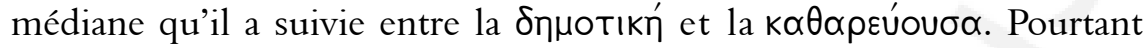
son amour pour la langue et la littérature grecques l'ont mené à publier plus de quarante volumes d'œuvres d'auteurs grecs, parmi lesquelles se trouvent celles d'Homère, d'Hippocrate, des orateurs attiques, les romans de Longus et d'Héliodore, et les Vies Parallèles de Plutarque. C'est grâce à une conférence de G. A. Christodoulou, publiée dans les actes du colloque international "Kopañs kai Xíos" qui a eu lieu à Chios en 1983, que nous avons découvert les Animadversiones in Luciani opera Editionis Bipontince. Il s'agit d'une section de notes de Coray, regroupées sous le titre Observationes Miscellanex (manuscrit n ${ }^{\circ} 490$ de la Bibliothèque "Adamantios Coray" de Chios), qui comptent 998 pages (disperses ainsi qu'en cahiers de douze pages) et qui contiennent plutôt des corrections de texte que des notes que Coray aurait prises pendant ses lectures de plus de soixante auteurs grecs. Toutes les feuilles sont numérotées par le même Coray. ${ }^{2}$

Les Animadversiones in Lucianum occupent 122 pages de $24 \times 18$ $\mathrm{cm}$, réunies en six cahiers (XX-XXV, p. 455-576 de la section sept du manuscrit); elles sont écrites à l'encre très lisiblement, et en latin. Elles concernent l'édition de Deux-Ponts (1789-1793), une simple réédition en dix volumes de l'édition de Reitz-Hemsterhuys de 1743, avec la traduction latine et les commentaires et variantes de la dite édition. ${ }^{3}$ Coray a noté plus de sept cent corrections du texte lucianesque, dont la plupart avaient déjà été proposées par d'autres philologues et quelquesunes ont été adoptées par les éditions modernes des œuvres de Lucien. Néanmoins, aucun éditeur moderne de Lucien n'a connu les corrections de Coray. Seul Macleod adopte deux corrections dans Toxaris ou sur l'amitié 9 et l'apocryphe Néron ou le percement de l'Isthme 10 et en mentionne une autre dans les Dialogues des morts 3.1. Une édition des Animadversiones in Lucianum montrerait le génie éditorial de Coray puisque elle prouverait que certaines corrections du texte de Lucien qui ont été adoptées aujourd'hui avaient été proposées par Coray il y a 200 ans.

\footnotetext{
${ }^{2}$ Cf. Christodoulou, op. cit., p. 37-53 (réimpr. dans son $\Sigma u ́ \mu \mu ı \kappa \tau \alpha K \rho ı \tau ı k \alpha ́$. Athènes: Édition privée, 1986, p. 237-255); cf. aussi Christodoulou, op. cit., les corrections de Coray sur Pindare (p. 94-96), Anacréon (p. 129-130) et Platon (p. 280-331), Bazou, op. cit., p. 465-480, mais surtout Kalospyros, op. cit., 2006.
}

${ }^{3}$ Cf. Bompaire, op. cit., 1993, p. CXXX-CXXXI (vol. I). 
Dans notre article, nous ne nous occuperons pas de toutes les corrections faites par Coray sur le texte de Lucien mais uniquement de celles qui n'ont été proposées par aucun philologue ou éditeur de Lucien jusqu'à présent. ${ }^{4}$ Notre présentation ne suivra pas non plus l'ordre du manuscrit de Coray, c'est-à-dire la série des opuscules lucianesques dans l'édition Bipontine, mais l'édition de Lucien dans les Oxford Classical Texts, réalisée par M. D. Macleod. ${ }^{5}$ D'abord nous donnons le texte de l'OCT avec le titre abrégé de l'œuvre de Lucien et le paragraphe où il se trouve, ainsi que le numéro du volume, de la page et de la ligne exacte de l'édition de Macleod entre parenthèses. Ensuite nous donnons la correction de Coray avec le numéro de la page du manuscrit et notre propre commentaire sur la correction proposée. On conserve la ponctuation de Coray dans les passages qu'il cite car parfois elle éclaire le sens du texte.

1. V.H.II 30 (I.116.8)

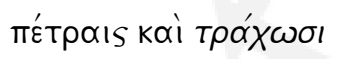

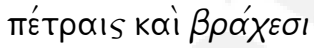

Coray corrige $\tau \rho \alpha ́ \chi \omega \sigma$ en $\beta \rho \alpha \alpha \chi \varepsilon \sigma$ (rupibus) et justifie sa proposition

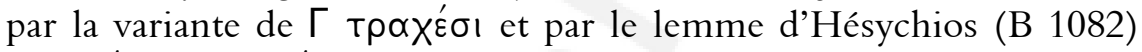

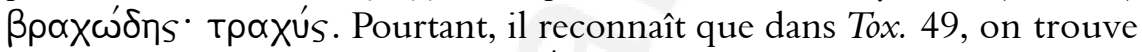

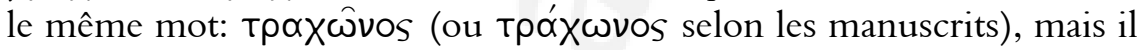
le considère comme regio scythica. Le pluriel $\beta \rho \alpha ́ \chi \varepsilon \alpha$ ("de l'eau peu profonde", synonyme du grec тєvó $\gamma \eta$ et du latin vada) est attesté chez Hérodote 2.102, 4.179 (bis), Thucydide 2.92.1, Diodore de Sicile 13.13.6, Arrien 24.2, 30.8, 38.7, 38.8, 39.7, 41.2 (bis), Polybe 1.39.3, 1.47.5, 1.51.11 et Plutarque, M. 980F. Néanmoins, il est peu probable qu'il existe une

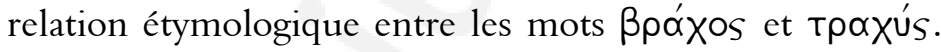

2. Cal. $23(\mathrm{I} .135 .26)$

(p. 521)

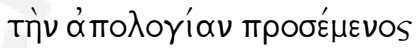

(Bekker: $\pi \rho o \alpha ı \sigma \theta o ́ \mu \varepsilon v o s$ codd.)

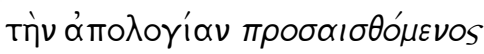

Coray connaît la leçon des manuscrits et aussi la correction de

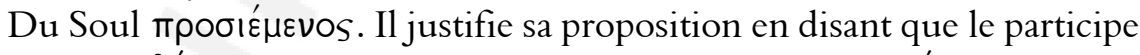

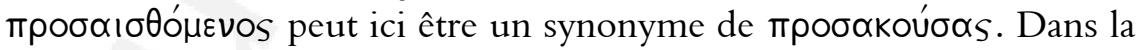

\footnotetext{
${ }^{4}$ Pour une liste complète des corrections de Coray du ms Chios 490 sur le texte de Lucien, cf. Kalospyros, op. cit., p. 87-105 (vol. II).

${ }^{5}$ Cf. Macleod, op. cit., 1972-1987. Infra nous nous référons aussi à d'autres éditions récentes, partielles ou d'ensemble.
} 
littérature classique, le verbe $\pi \rho \circ \sigma \alpha ı \sigma \theta \alpha ́ v o \mu \alpha ı$ n'est attesté qu'une seule fois chez Aristote, Mem. remin. 450a.21, et signifie "concevoir de plus". Bompaire cite la remarque "ingénieuse" de Belin pour le verbe

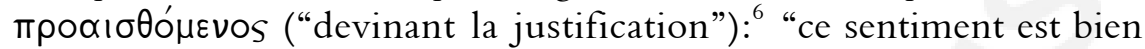
celui d'un caractère généreux; il est très délicat; les commentateurs n'en ont point senti la finesse". Bien que la version syriaque récemment découverte s'accorde avec la correction de Du Soul, Coray a conservé la leçon des manuscrits en remplaçant simplement le préfixe про- par проб-.

3. Cal. 24 (I.136.8)

(p. 521-522)

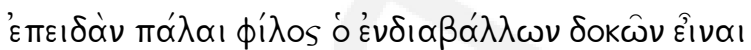

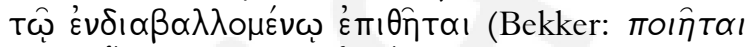

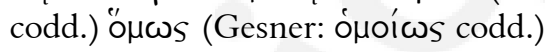

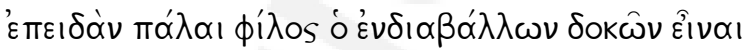

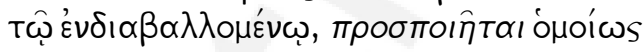

Coray ne pourrait pas connaître la correction de Bekker; la leçon des manuscrits lui paraît plus plausible et il propose la correction пробтоюñरа еn la justifiant par simulat pariter, qui est tout à fait en accord avec le contexte du passage. Bompaire et Jufresa-Mestre-Gómez n'ont pas adopté la correction de Bekker et impriment noı̄̄T ${ }^{7}{ }^{7}$ tandis

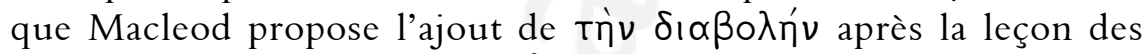

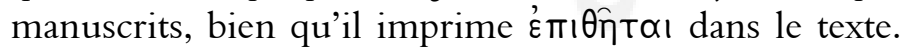

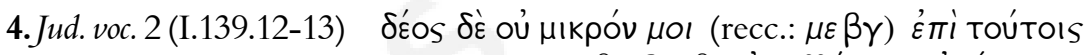
(p. 456)

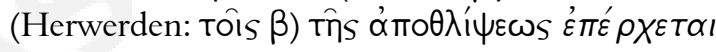

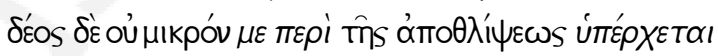

Macleod suit partiellement Herwerden qui a corrigé le tôs des

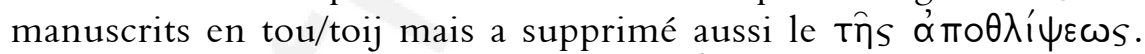
Bompaire a simplement supprimé le Tôs, ${ }^{8}$ tandis que Mestre-Gómez

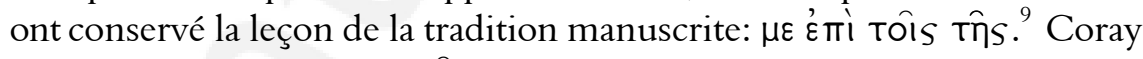
supprime également le tốs et corrige non seulement le préfixe du verbe $\varepsilon \pi \varepsilon^{\prime} \rho \chi \varepsilon \tau \alpha$, mais aussi le datif de l'objet du verbe $\mu$ o. Il justifie

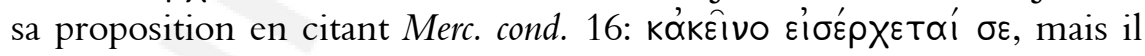
reconnaît que dans l'apocryphe Alc. 3, nous trouvons le même verbe

${ }^{6}$ Cf. Bompaire, op. cit., p. 163 (vol. II, n. 59) et Jufresa, Mestre et Gómez, op. cit., p. 97.

${ }^{7}$ Cf. Bompaire, op. cit., p. 164 (n. 64).

${ }^{8}$ Cf. Bompaire, op. cit., p. 179.

${ }^{9}$ Cf. Mestre et Gómez, op. cit., p. 70. 


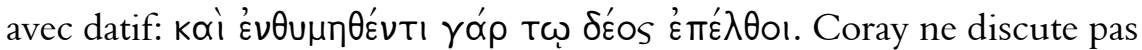
l'authenticité de L'alcyon ou sur les métamorphoses; pour lui, il s'agit d'un parallèle lucianesque. Dans la littérature grecque, nous trouvons le verbe

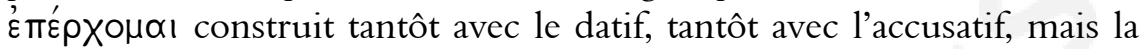

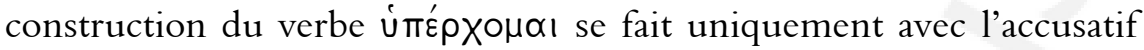
excepté chez Plutarque, Comp. Pericl. et Fab. Max. 2.2. Coray corrige

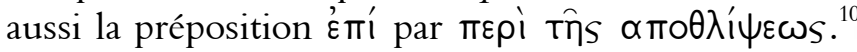
5. Cat. 19 (I.193.9)

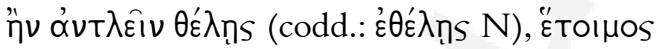
(p. 468)

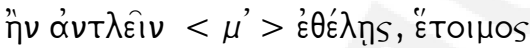

Cette correction semble insignifiante, mais ce passage a fait beaucoup réfléchir les éditeurs de Lucien: Macleod et Bompaire ${ }^{11}$ suivent l'ordre des mots que donnent les manuscrits mais ils choisissent

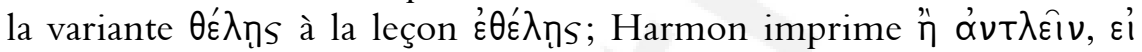
$\theta \dot{\varepsilon} \lambda \eta \emptyset s$, हैтoıнos, tandis que Fritzsche préfere changer l'ordre des mots

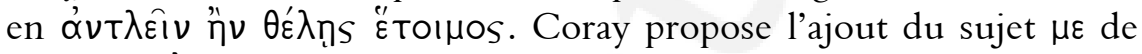
l'infinitif $\alpha$ ó $\nu \tau \lambda \varepsilon \hat{\imath} \nu$, une correction simple mais tout à fait conforme au contexte: "si tu veux que j'écope, je suis disponible". Nous trouvons

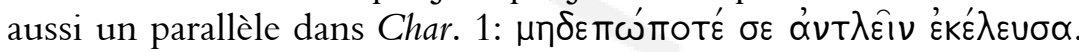
6.J. trag. 44 (I.243.14)

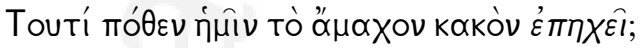

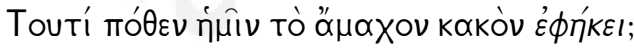

Les manuscrits sont divisés quant au verbe principal de cette

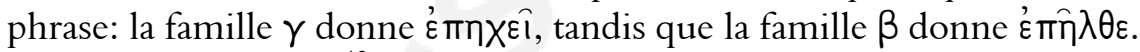
Macleod et Coenen ${ }^{12}$ préferent la première leçon, mais Harmon l'a

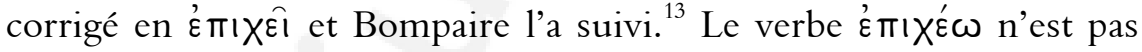
inconnu de Lucien; nous le trouvons aussi dans Luct. 19, Nav. 44, et dans le même J. trag. 35. Pourtant Coray propose le verbe غ̇ chez Sophocle, Ant. 1257, Aj. 34 et El. 304, Hippocrate, Nat. hom. 1, Thucydide 8.67.2, Xénophon, Rsp. Lac. 12.5, et Pausanias 8.40.3. Coray

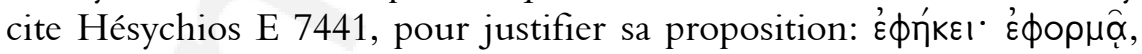

\footnotetext{
${ }^{10}$ Dans la p. 477 (corr. no 72, cf. infra) il cite le passage de nouveau avec pour seule

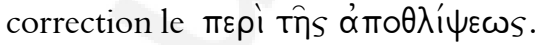

${ }^{11}$ Cf. Bompaire, op. cit., p. 287.

${ }^{12}$ Cf. Coenen, op. cit., p. 28.

${ }^{13}$ Cf. Bompaire, op. cit., p. 73 (vol. III). Pour une discussion sur le passage, aussi Nesselrath, H. G., c.r. de Macleod, M. D. Luciani Opera. Vols. I-III. Oxford: Oxford University Press, 1972-1980 (Gnomon, Munich/Berlin, vol. 56, p. 607, 1984).
} 


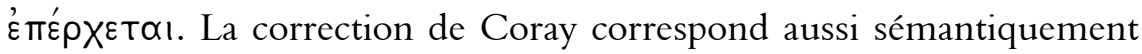
à la leçon de la famille $\beta$.

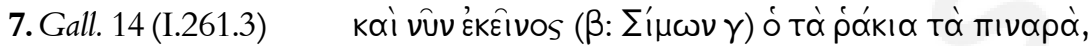
(p. 508)

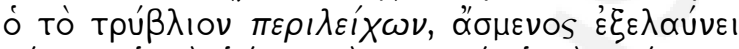

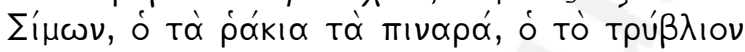

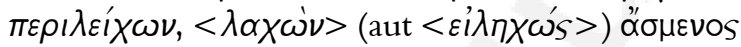

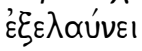

Il s'agit d'une correction de compréhension du texte. Comme signale Bompaire "ơ $\sigma \mu \varepsilon v o s$ est parfois rattaché à $\pi \varepsilon \rho ı \lambda \varepsilon i ́ \chi \omega \nu$ " ${ }^{14}$ Coray ne semble pas convaincu par cette explication. Il connait les discussions des philologues pour ce passage, discussions qui n'ont néanmois pas donné de solution. C'est pourquoi il fait une autre proposition: il introduit le participe $\lambda \propto \chi \omega^{\prime} \nu$ ou $\varepsilon i \lambda \eta \chi \omega^{\prime}$ juste après $\pi \varepsilon \rho ı \varepsilon^{\prime} \mid \chi \omega \nu$, un mot qui ressemble paléographiquement au second terme du verbe, mais qui se réfere syntaxiquement au Tà ṕókı $\alpha$. Ainsi il ajoute une caractéristique de plus à Simon, la personne dont on parle dans ce passage.

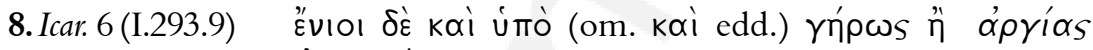
(p. 509)

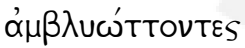

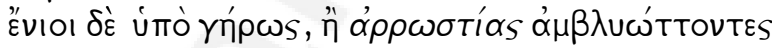

Dans ce passage, Coray pense que le ópría ne peut pas causer un mal aux yeux. Il note qu'ici il fallait dire quelque chose comme åp $\rho \varepsilon \mu o v$, "le glaucome", ou ơ $\lambda \gamma \varepsilon$ ín qui signifie "maladie" en général, selon Hésychios A2797. Cela justifierait le verbe $\alpha \mu \beta \lambda \cup \omega ́ \tau T \omega$, "avoir la vue faible", et aussi la présence de la vieillesse dans la même phrase. Alors il corrige en

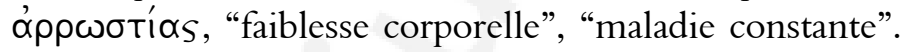

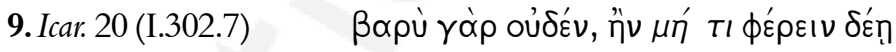

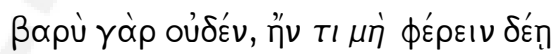

Coray observe qu'avec cette minime transposition de mots, qui ne change pas le sens $\mathrm{du}$ texte, on construit un trimètre iambique ${ }^{15}$ (perfectus senarius) que Lucien pourrait avoir emprunté à un poète

\footnotetext{
${ }^{14}$ Cf. Bompaire, op. cit., p. 124 (n. 55).

${ }^{15}$ Le Professeur H. G. Nesselrath m'a désigné avec juste raison que ce trimètre n'est pas correct car la dernière syllabe de $\beta \propto \rho u ́$ est toujours brève et il m'a indiqué les passages suivants: Homère, Od. 8.95, 8.534, Euripide, Hel. 268, Ph. 1641, Apollonios de Rhodes 4.1492, Anthologie Grecque 2.182, 6.220.11, 6.290.3, 9.310.3, 16.133.9, Nonnos, Dion. 1.38, 6.51, 19.1, 19.8 etc, Quintus de Smyrne 1.374, 2.167, 2.660 etc.
} 
comique. Mettre dans la bouche des personnages des vers iambiques est une pratique très courante chez Lucien. ${ }^{16}$

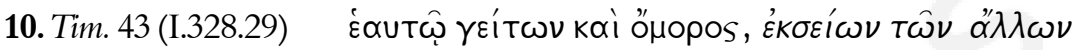
(p. 457)

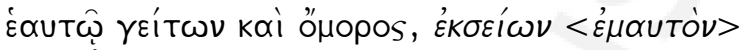
$\tau \hat{\omega} \nu \alpha^{\prime \prime} \lambda \lambda \omega \nu$

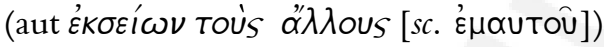

Ce passage a posé beaucoup de problèmes aux philologues: dans

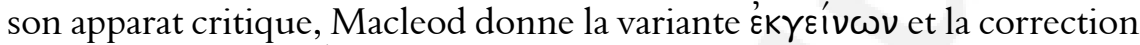

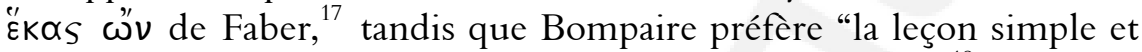

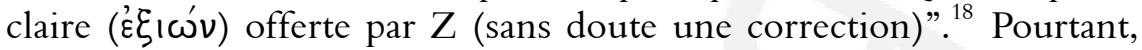
toutes les objections des philologues portent sur le génitif qui suit le verbe $\varepsilon k \sigma \varepsilon i ́ \omega \nu$ qui devrait ainsi être intransitif: par conséquent,

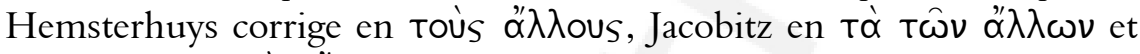
Benedictus en Tòv $\alpha$ $\alpha \lambda$ ov. Coray, à son tour, fait plusieurs propositions:

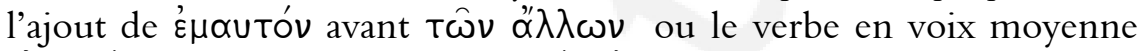

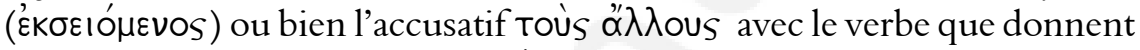

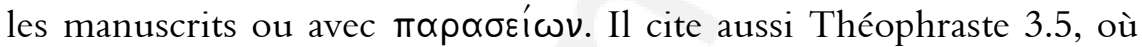
$\pi \propto \rho \propto \sigma \varepsilon i \omega$ et $\alpha \dot{\alpha} \alpha \lambda \lambda \lambda \alpha^{\prime} т \tau о \mu \alpha$ । voisinent.

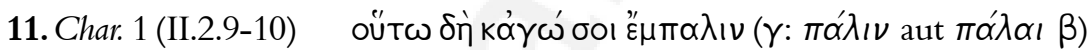
(p. 463) $\dot{\alpha} \mu \beta \lambda \cup \omega \dot{\tau} \tau \omega$ про̀ sò $\phi \omega \hat{s}$

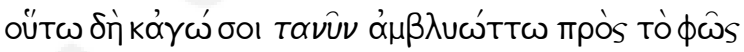

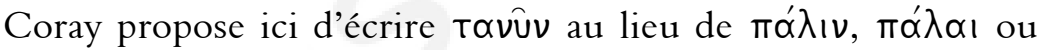
$\varepsilon \mu \mu \pi \alpha \iota \nu$ des manuscrits. Cela peut sembler une correction peu nécessaire mais le passage a suscité bien des débats chez les philologues. L'expression $\tau \grave{\alpha} \nu \hat{v} \nu^{19}$ (en deux mots) n'est pas inconnue de Lucien; on la trouve quatre fois dans son ouvre: Dear. jud. 12, Dem. enc. 48, Scyth. 9 et D. meretr. 9.4.

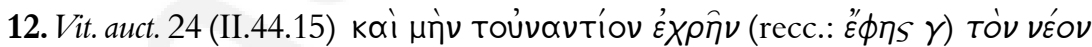
(p. 466)

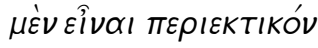

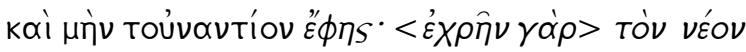

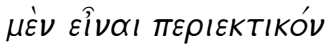

\footnotetext{
${ }^{16}$ Cf. Karavas, op. cit., 2005, p. 137-170.

${ }^{17}$ Cf. Hopkinson, op. cit., p. 60, imprime" $\varepsilon \chi \propto \varsigma \varsigma$ $\omega " \nu$.

${ }^{18}$ Cf. Bompaire, op. cit., p. 317 (n. 92).

${ }^{19}$ Kalospyros transcrit ainsi la correction de Coray [op. cit., p. 104 (vol. II)]
} 
Coray fait une combinaison des différentes leçons des manuscrits

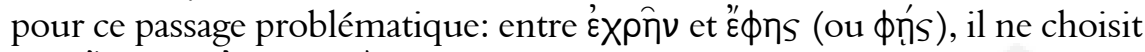

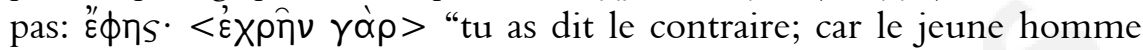
devrait recevoir". Néanmoins, il devient même plus audacieux en

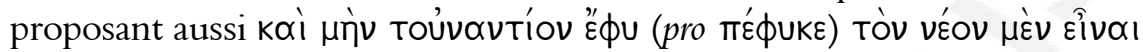
пєрієктIKóv, "mais au contraire, par nature le jeune homme reçoit".

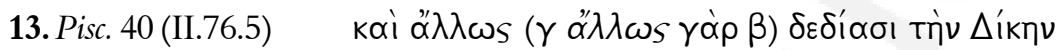
(p. 468)

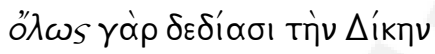

Coray ne donne pas d'explication sur cette correction qu'il propose: tout simplement, il trouve $\alpha^{\prime} \lambda \lambda \omega s$ douteux et le change en

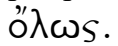

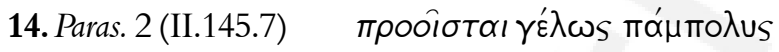

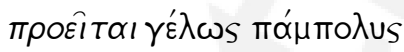

Bien que la tradition manuscrite transmette ici unanimement la troisième personne du singulier du parfait de la voix passive du verbe трофє́рона। - une attestation unique dans la littérature grecque -, les philologues sont intervenus de plusieurs manières: Madvig corrige en

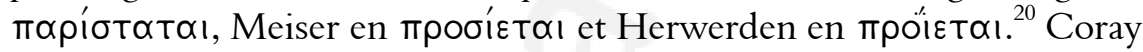

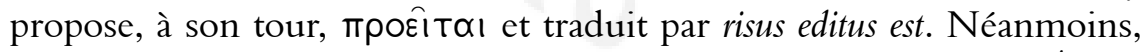

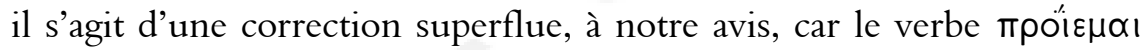
est presque toujours transitif au moyen et non pas passif et signifie "lancer", "jeter", "abandonner".

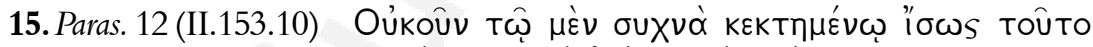
(p. 512)

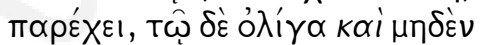

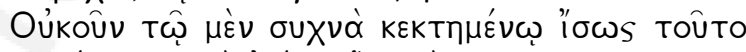

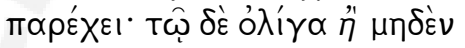

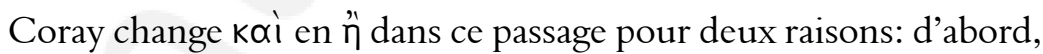
il donne un texte parallèle chez Lucien, quelques paragraphes plus haut, qu'il trouve en rapport avec celui-ci, où les deux termes de comparaison

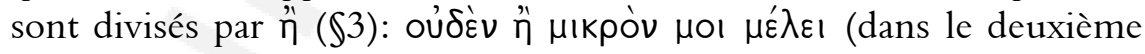

${ }^{20}$ Pour toutes les corrections proposées, cf. Nesselrath, op. cit., 1985, p. 266. Nesselrath l'obélise: †троốтаı† (op. cit., 1985, p. 497).

${ }^{21}$ Cf., e.g. chez Lucien, V.H.I. 8, 29, V.H.II. 40, 46, Icar. 20, Dem. enc. 44. Cf. Libanios, Ep.

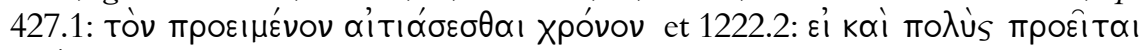
xpóvos. 


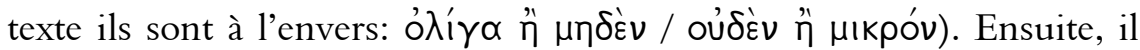
interprète $\pi \alpha \rho{ }^{\prime} \chi \varepsilon \mid$ par ${ }^{\prime \prime} \xi_{\varepsilon \sigma T I}$ (licet) pour éclairer le passage, comme l'utilisent Hérodote $(1.9,1.170,3.73,3.142$, 4.140, 5.98, 8.140) et Hippocrate $(\mathrm{Sem} .7,34)$. Le verbe en question a été mis en doute par la tradition manuscrite car on y trouve la variante úmápXєı.

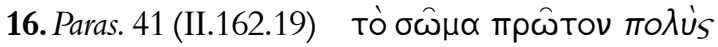

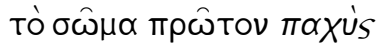

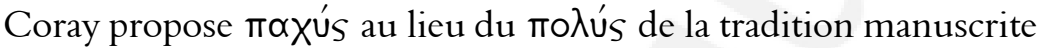
car, quelques lignes plus haut, Lucien parle de $\lambda \varepsilon \pi$ Toús (\$40) et suppose qu'il fallait montrer ici le contraste. ${ }^{22}$ Coray a peut-être raison puisque Lucien oppose $\pi \propto x u ́ s$ à $\lambda \varepsilon \pi T o ́ s$ deux fois dans son ouvre: Salt. 76 et D. meretr. 14.3.

17. Paras. 42 (II.163.4) (p. 513)

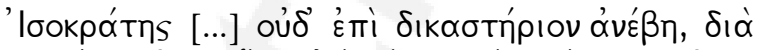

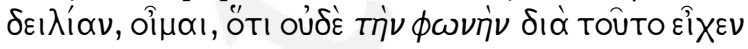

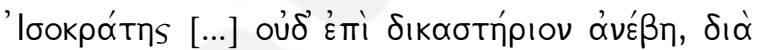

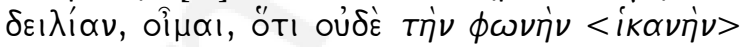

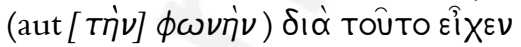

Coray commence par qualifier le texte d'"étrange" (locus est rarus) puis

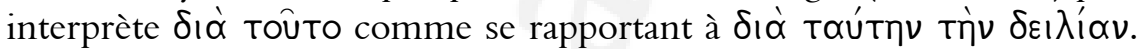
Dindorf avait déjà corrigé $\delta ı \alpha$ toûto par $\delta ı \alpha \rho k o u ̂ \sigma \alpha \nu$ (se référant

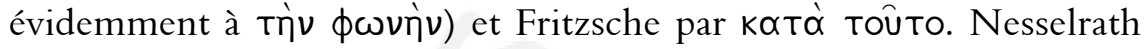
fait un très bon commentaire du texte en citant des passages d'Isocrate et d'autres auteurs qui parlent de lui, et supprime ótı en ajoutant un attribut après $\varepsilon \hat{\imath} X \varepsilon v .^{23}$ Coray, à son tour, cite deux passages d'Isocrate, où

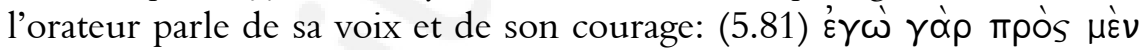

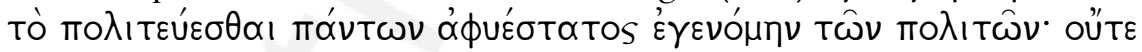

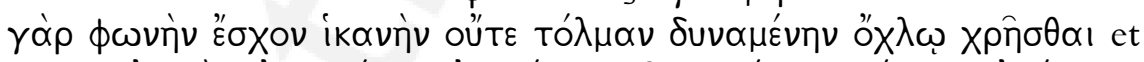

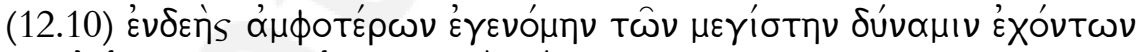

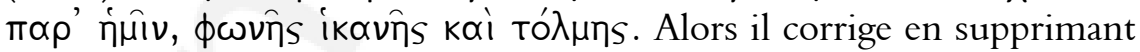
l'article тńv et en laissant $\phi \omega v \eta \dot{v} v$ seul, ou bien en ajoutant l'épithète íavív, mot-clé dans les fragments rhétoriques qu'il vient de citer.

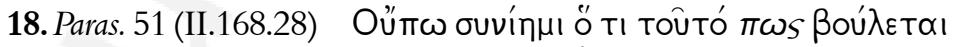
(p. 515)

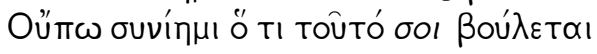

\footnotetext{
${ }^{22}$ Nesselrath, op. cit., 1985, p. 405-406 ne trouve ici aucun problème textuel ou sémantique.

${ }^{23}$ Cf. Nesselrath, op. cit., 1985, p. 426-427 et p. 498.
} 
Certe illud $\pi \omega s$ abundare videtur, dit Coray, sans plus d'explications; et il le change en $\sigma 0 .^{24} \mathrm{La}$ famille $\gamma$ donne $\pi \hat{\omega} s$.

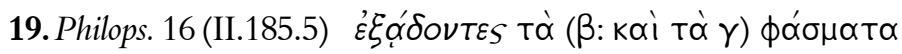
(p. 518)

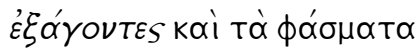

Coray explique cette nouvelle correction en citant un passage qui se

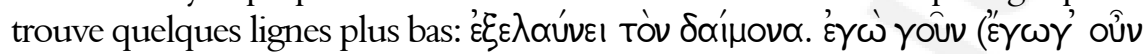

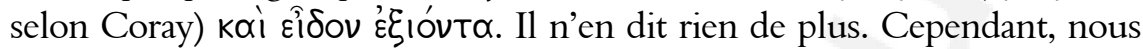

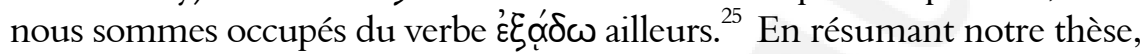
nous répétons que $\varepsilon \xi \hat{q}^{\prime} \delta \omega$ est utilisé par Lucien deux fois seulement dans son œuvre pour se référer exclusivement à Jésus-Christ. C'est pourquoi nous croyons que la proposition de Coray ici n'est pas nécessaire.

20. Dear.jud. 3 (II.202.15) (p. 459)

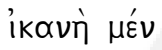
$\kappa \alpha \lambda \eta \dot{\mu} \mu \varepsilon^{\prime} v$

Il s'agit d'une correction de compréhension du texte. On parle de l'amante de Pâris. Coray préfere lire ici que la femme est $k \alpha \lambda \eta ́$ (pulchra) au lieu de ikavń. Il avoue aussi qu'il s'est inspiré de Hemsterhuys. Cependant, sa proposition semble peu nécessaire car le principal argument d'Aphrodite sur la belle Hélène est justement qu'elle est belle (paragraphes 13-14: sa mère aussi). Si Pâris avait déjà une amante belle, pourquoi en chercher une autre qui le soit aussi?

21. Dear.jud. 7 (II.205.21) (p. 460)

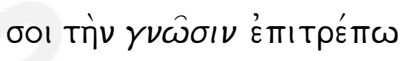

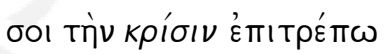

Encore une correction de compréhension du texte. Ici Coray semble avoir raison: mis à part le titre de l'opuscule, $\Theta \varepsilon \hat{\omega} \nu$ kpíols, le mot $\gamma v \omega \hat{\sigma} / \mathrm{s}$ n'y est attesté qu'une seule fois en regard des six occurrences de kpíols et kpíveılv qui sont répétés toujours en référence à la capacité de Pâris de juger.

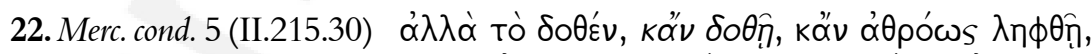
(p. 469)

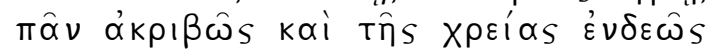

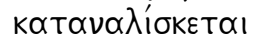

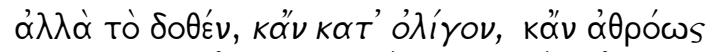

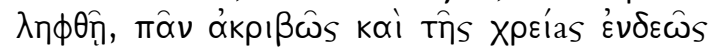

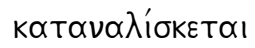

\footnotetext{
${ }^{24}$ Dans ses notes marginales sur ce texte, Coray propose по .

${ }^{25}$ Cf. Karavas, op. cit., 2010, p. 119-120.
} 
Coray estime que le texte est douteux (locus suspectus); il pense que $\delta \circ \theta \eta ̣$ a été ici redoublé par erreur à cause du $\delta \circ \theta^{\prime} v$ qui précède. Il cite aussi

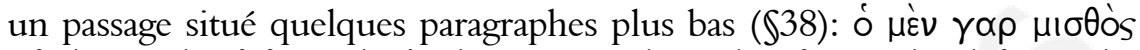

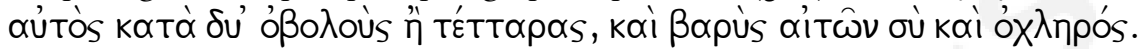

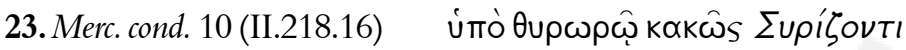
uं

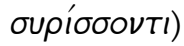

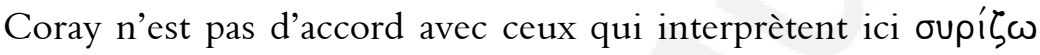
par "parler comme un Syrien"; il pense que Lucien décrit plutôt une image qui survit jusqu'à nos jours: le concierge qui siffle!

24. Merc. cond. 26 (II.227.15) (p. 471)

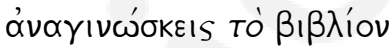

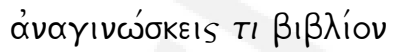

Encore une correction sans explication de la part de Coray.

25. Merc. cond. 26 (II.227.25) (p. 471)

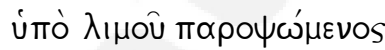

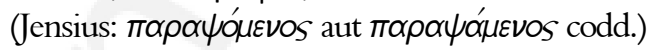

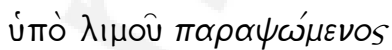

Coray ne connaît pas la correction $\pi \propto \rho \circ \psi \omega \dot{\mu \varepsilon v o s ~ d e ~ J e n s i u s, ~ a l o r s ~}$ il propose la sienne, $\pi \alpha \rho \alpha \psi \omega \dot{\mu \varepsilon v o s ~(a b r a d e n s): ~ i l ~ t r a d u i t ~ l e ~ v e r b e ~}$

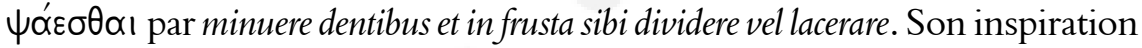
est Hésychios ( 460$): \psi \alpha \alpha \mu \mu \alpha \tau \alpha \cdot \sigma \pi \alpha \rho \alpha ́ \gamma \mu \alpha \tau \alpha$. C'est vrai que le verbe

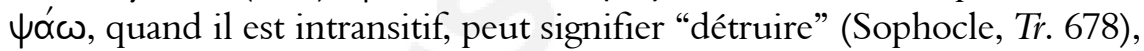

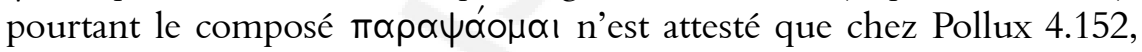
deux fois, et se réfere au cheveu, au sens de "lisser".

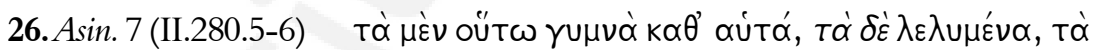
(p. 502) $\delta \varepsilon \dot{\varepsilon} \sigma T \varepsilon \phi \alpha^{\prime} v O I S \pi \varepsilon \pi \lambda \varepsilon \gamma \mu \varepsilon^{\prime} v \alpha$

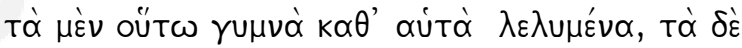

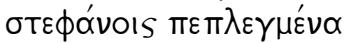

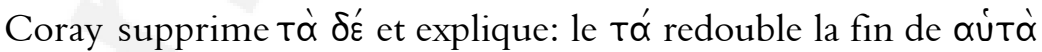
et le $\delta^{\prime} \varepsilon$, le début de $\lambda \varepsilon \lambda u \mu \varepsilon \dot{v} \alpha$.

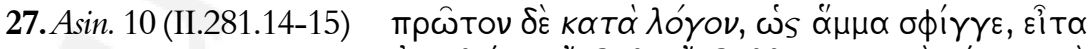
(p. 503)

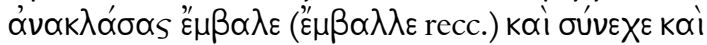

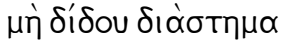

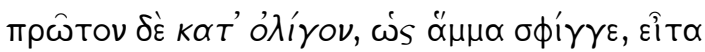

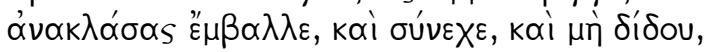
$\delta ı \alpha ั \sigma т \eta \mu \alpha$ 
Coray justifie cette nouvelle correction en citant un passage situé

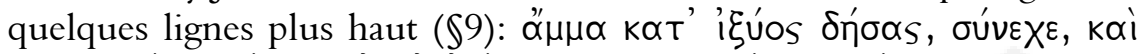

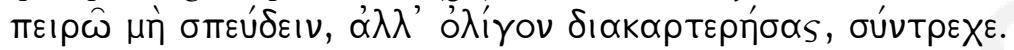

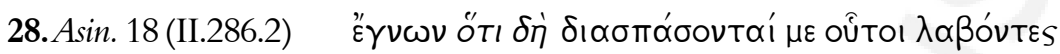
(p. 503)

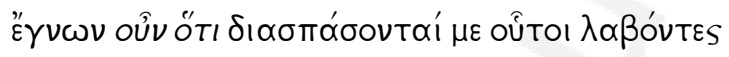

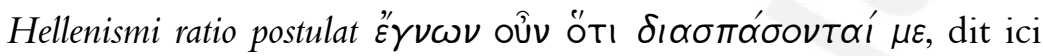
Coray, qui explique que le oûv a été absorbé par la fin de $\varepsilon^{\prime} \gamma \nu \omega \nu$ et le $\delta \eta ́$

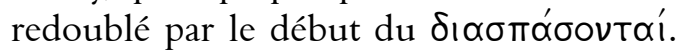

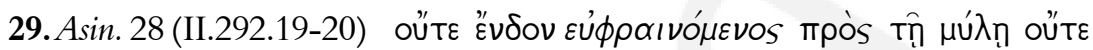
(p. 504)

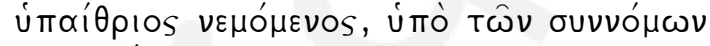

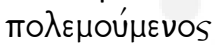

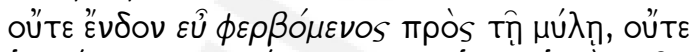

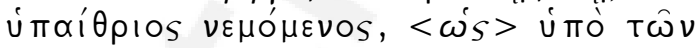

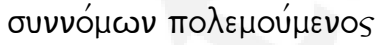

La correction importante ici pour Coray est le $\omega_{s}$, qui a été

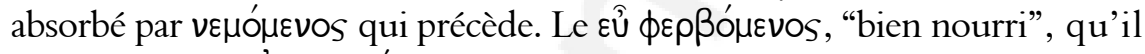

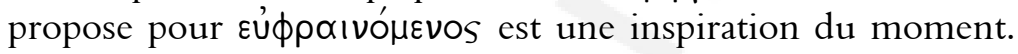

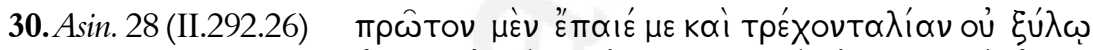
(p. 504)

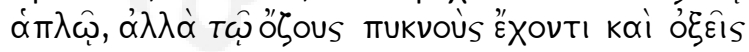

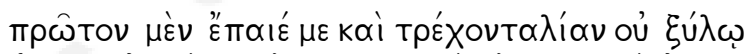

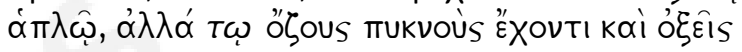

Nous présentons cette correction minime, bien qu'elle soit aussi proposée par Nesselrath, ${ }^{26}$ car nous la trouvons tout à fait correcte: au lieu de l'article défini $\tau \hat{\omega}$ qui se réfere au $\xi u ́ \lambda \omega$, avec lequel le patron de l'âne le battait, Coray propose le pronom tழ̣ avec juste raison. Le patron n'avait pas plusieurs bâtons parmi lesquels il aurait choisi celui fait de nœuds pointus pour frapper Lucius, mais il s'agissait d'un bâton quelconque.

31. Asin. 30 (II.292.9-11) (p. 505)

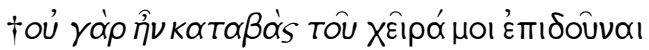

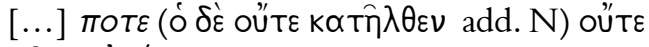

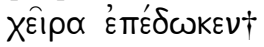

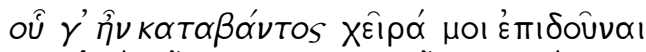

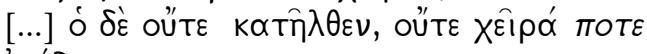
$\varepsilon \pi \varepsilon \delta \omega K \varepsilon \nu$

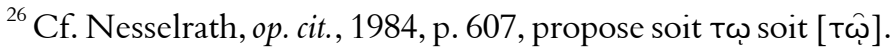


Coray ne donne pas de solution sur ce texte corrompu. Tout simplement, il propose la correction qui paraît la plus proche de la leçon que donnent les manuscrits. ${ }^{27}$

32. Asin. 40 (II.298.28)

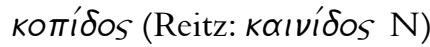
kaıvíos

Macleod imprime $k \alpha ı$ ííos dans l'édition Loeb, ${ }^{28}$ mais sept années plus tard, dans l'édition OCT, il adopte la correction de Reitz

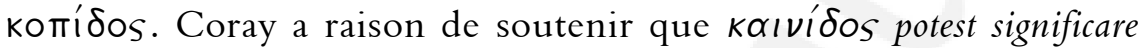

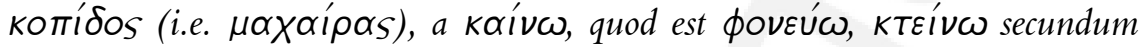
Hesychium (K239); il ajoute que, dans la langue française, kaıvís a donné

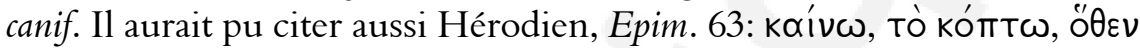

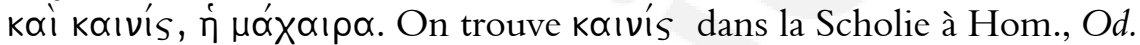
24.230 et dans la Scholie à Eur., Or. 1302.

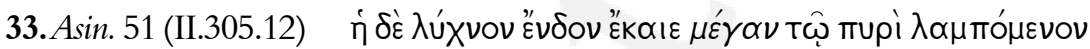
(p. 505)

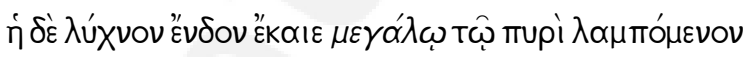

Il s'agit d'une correction de style; Coray trouve beaucoup plus

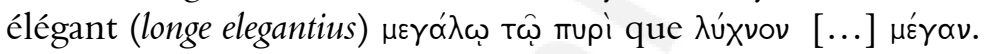

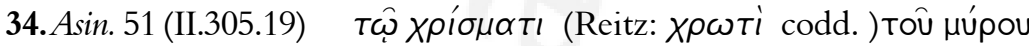
(p. 506)

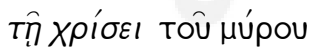

La faute dans ce passage, selon Coray, porte sur l'interprétation

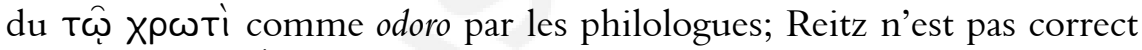
non plus car $\chi \rho i \sigma \mu \alpha T I$ se trouve aussi au $\$ 54$ au sens de unguenti, c'est-

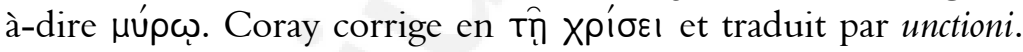

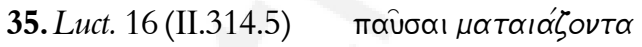
(p. 516)

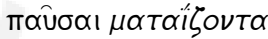

Coray propose ici une forme différente du même verbe $\mu \alpha \tau \alpha \underline{\zeta} \zeta \omega$ : $\mu \alpha \tau \alpha i \zeta \omega$ au lieu du $\mu \alpha \tau \alpha ı \alpha ́ \zeta \omega$ que donne la tradition manuscrite. Eustathe de Thessalonique (Il. 2.64) atteste que les deux formes sont courantes. ${ }^{29}$ Néanmoins Coray a peut-être bien deviné; dans la Suda

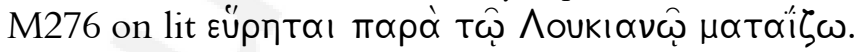

\footnotetext{
${ }^{27}$ Cf. Nesselrath, op. cit., 1984, p. 606.

${ }^{28}$ Cf. Macleod, op. cit., 1967.

${ }^{29}$ Cf. Kambitsis, op. cit., p. 44.
} 
36. Rh.pr. 4 (II.318.18)

n̈ ràp 'Hoíodos

(p. 517)

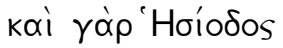

Selon l'apparat critique de Macleod, $\dddot{\eta}$ est une correction de Sommerbrodt; ${ }^{30}$ selon Coray, de Guyet. De toute façon, la tradition manuscrite donne eí. Il y a aussi la proposition de Sauppe tí róp; 'Hoíodos. Coray corrige en kai sans donner plus d'explications.

37. Rh.pr. 10 (II.322.15-16) (p. 517)

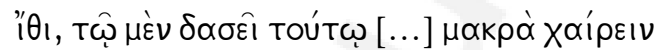

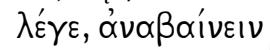

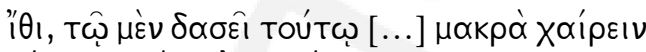
$\lambda \varepsilon^{\prime} \gamma \varepsilon,<k \alpha i>\alpha^{\prime} \nu \alpha \beta \alpha i v \varepsilon ı$

Coray ajoute k $\alpha i$ ici et explique que la construction de la phrase

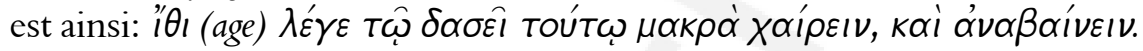

38. Alex. 23 (II.342.19) (p. 488)

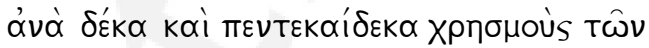

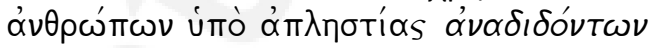

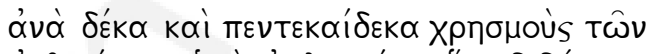

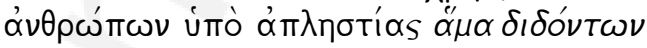

Selon Coray, la tradition manuscrite donne la variante $\alpha_{\mu \alpha} \alpha \delta^{\prime} \varepsilon \alpha$

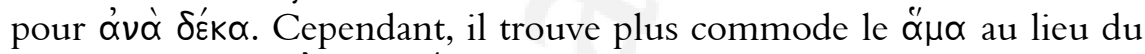

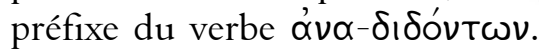

39. Alex. 48 (II.352.24) "̈x (p. 489)

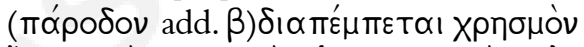

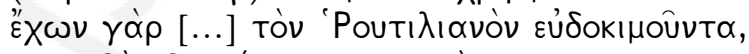

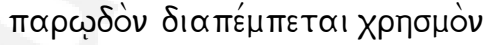

Coray conserve la leçon mápoঠov de la tradition manuscrite que

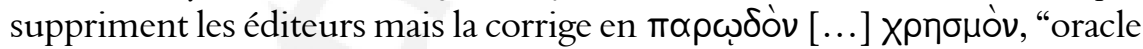
allusif", et donne comme parallèle un texte d'Euripide, $I A$ 1146-1147:

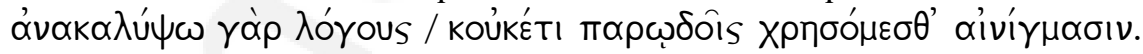

40. Syr. dea 30 (III.16.16-17) (p. 542)

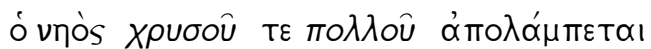

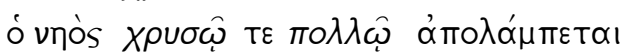

Encore une correction de style; Coray trouve beaucoup plus

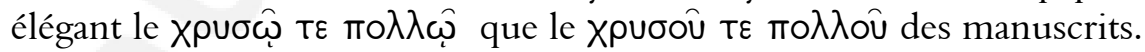
Pourtant, Lightfoot offre de bons arguments pour le génitif du texte et

${ }^{30}$ Cf. aussi Zweimüller, op. cit., p. 193.

${ }^{31}$ Dans ses notes marginales sur ce texte, Coray propose $\hat{\eta}^{\circ}$ 
aussi pour la voix moyenne $\alpha^{\prime} \pi 0 \lambda \alpha^{\prime} \mu \pi \varepsilon \tau \alpha \iota_{1}{ }^{32}$ La tradition manuscrite n’y donne pas de variante.

41. Syr. dea 51 (III.22.28) (p. 542)

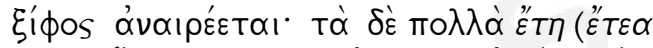

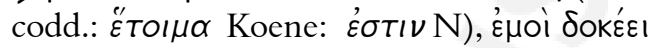

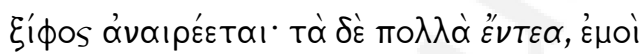

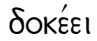

"A puzzling sentence", avoue Lightfoot, ${ }^{33}$ Coray connaît toutes

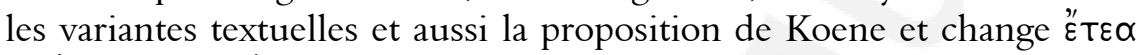

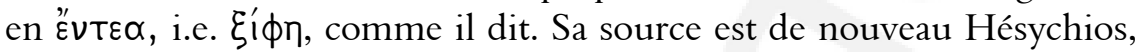

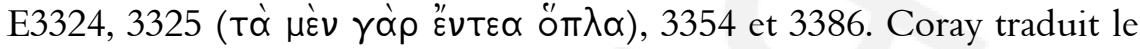
singulier हैvTos par ensis.

42. Syr. dea 60 (III.25.1-2) (p. 543)

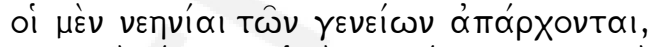

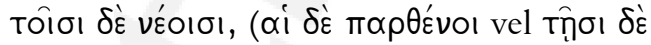

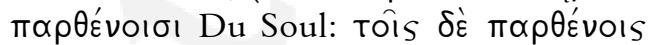

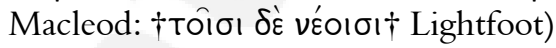

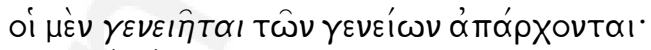

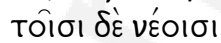

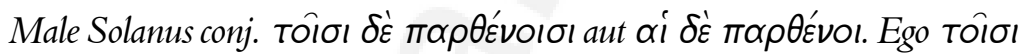
véoı latere suspicor, pro quo forsan scripsit Lucianus ГENHÏHTAI (Jonice) aut ГENEIHTAI, barbati qui optime opp. Tôls véols i.e. Tôs áreveíols. Le

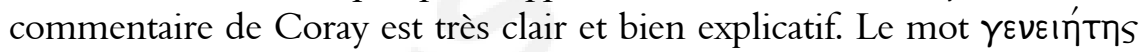
n'est pas inconnu de Lucien (Bis acc. 28, Sacr. 11, Rh. pr. 23 et même Syr.

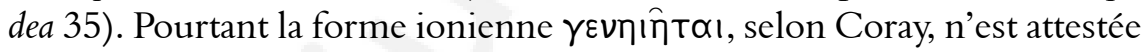
qu'une seule fois dans la Chron. 282 de Malalas, quelques siècles plus tard. Coray a bien localisé le problème de sens dans vequíaı et le corrige

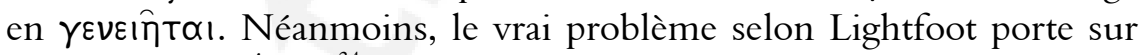
le datif Tốı véoı ${ }^{34}{ }^{34}$

43. Lex. 6 (III.60.4-5)

(p. 493)

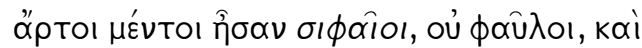

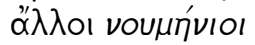

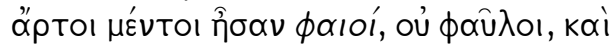

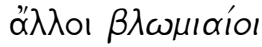

\footnotetext{
${ }^{32}$ Cf. Lightfoot, op. cit., p. 432.

${ }^{33}$ Cf. Lightfoot, op. cit., p. 508.

${ }^{34}$ Cf. Lightfoot, op. cit., p. 536 (n. 20).
} 
La source de Coray pour ces deux corrections est Athénée 3.114D-

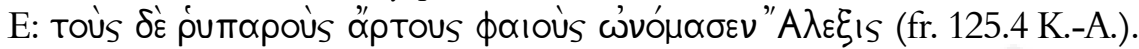

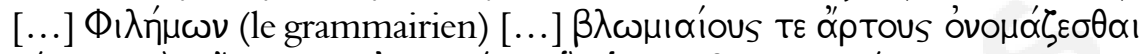

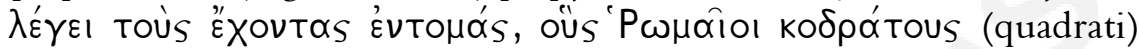

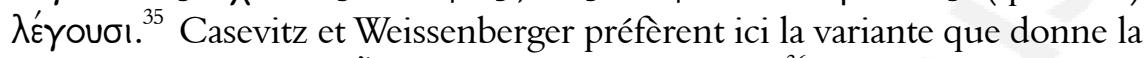

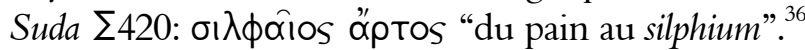

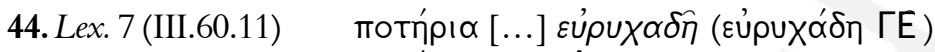
(p. 493)

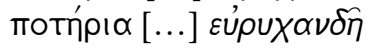

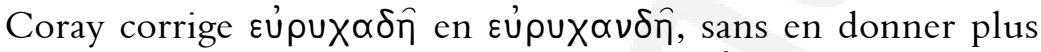

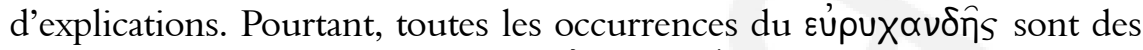

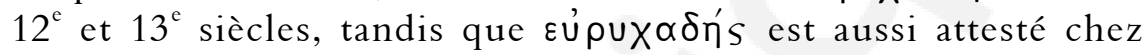
l'épigrammatiste Léonidas d'Alexandrie, $A P$ 6.305.4, et même comme épithète du $k u ́ \lambda_{\imath} \xi$. Néanmoins, Weissenberger attire notre attention sur

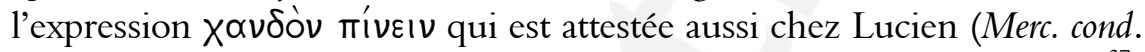
7, D. mort. 13.6, D. meretr. 6.3; cf. Pollux 6.25 et Hésychios X157-158). ${ }^{37}$

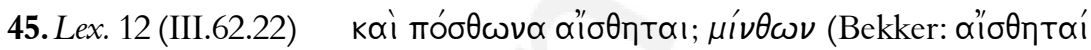
(p. 494)

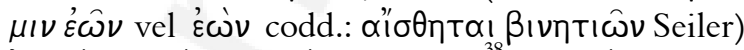

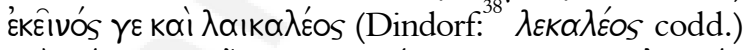

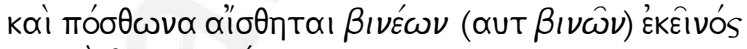

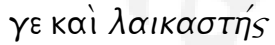

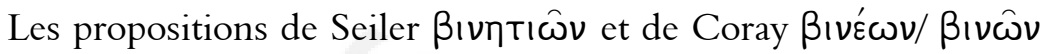
de $\mu I V \varepsilon \omega \nu$ de la tradition manuscrite sont tout à fait conformes au contexte sexuel et comique du passage. ${ }^{39}$ De même, la correction de Bekker $\mu \prime \nu \theta \omega \nu$ que suit Macleod contient un double sens, puisque

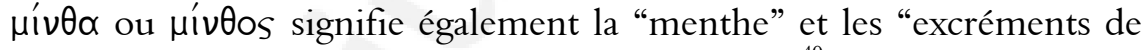
l'homme" (Hésychios M1393, \%309, Suda M1085). ${ }^{40}$ De plus, Hésychios

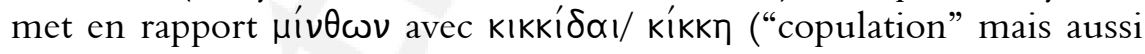

${ }^{35}$ Cf. García Soler, op. cit., p. 82-83 et p. 90.

${ }^{36}$ Cf. Casevitz, op. cit., p. 84 et Weissenberger, op. cit., p. 216-217. Sur le silphium dans la cuisine grecque, cf. García Soler, op. cit., p. 365-367.

${ }^{37}$ Cf. Weissenberger, op. cit., p. 223.

${ }^{38}$ Cf. Nesselrath, op. cit., 1984, p. 588.

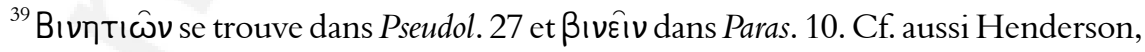
op. cit., p. 151-153 et Weissenberger, op. cit., p. 257.

${ }^{40}$ Cf. Henderson, op. cit., p. 185. Casevitz (op. cit., p. 84) traduit «un homme de menthe». 
"la mauvaise odeur des parties génitales"; K2651-2652). Mestre-Gómez

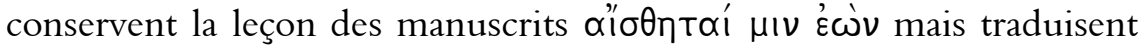
correctement en "inmundicia", c'est-à-dire "excréments". ${ }^{41}$ Coray

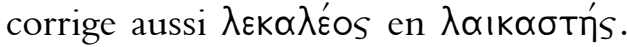

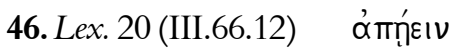

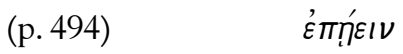

Aucune explication pour cette correction de la part de Coray.

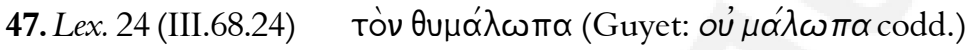

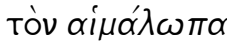

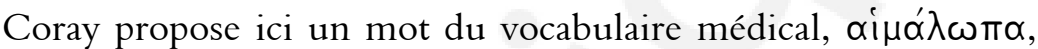
l'"hématome", sans en donner plus d'explications. Cependant, il semble

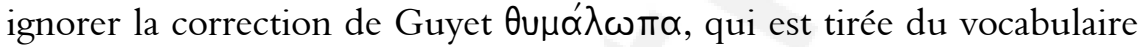
comique (Aristophane Th. 729, Ach. 321, Strattis fr. 58.2 K.-A.).

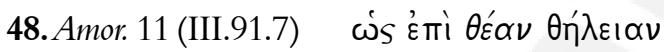

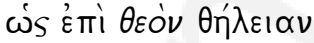

Coray est en désaccord avec les philologues qui interprètent le

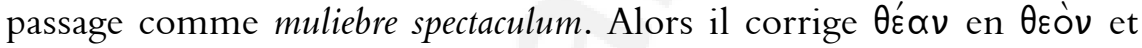
explique: tanquam ad femininum numen, nempe Venerem.

49. Amor. 14 (III.92.22) (p. 496)

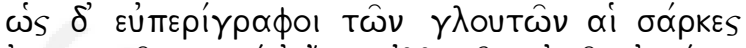

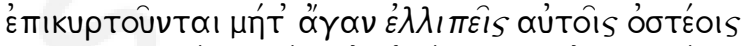

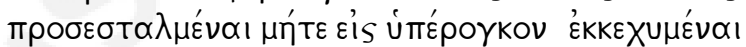

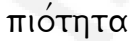

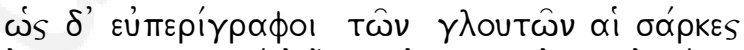

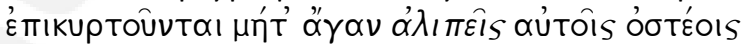

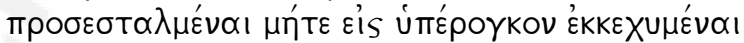

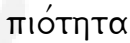

Selon Coray, la fin de la phrase exige de lire ici $\alpha \lambda_{1} \pi \varepsilon \hat{~}$ s. On

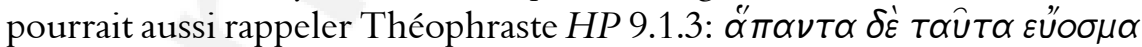

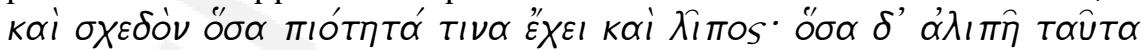
$\delta \alpha^{\prime \prime} \circ \sigma \mu \alpha$.

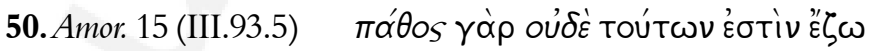
(p. 496)

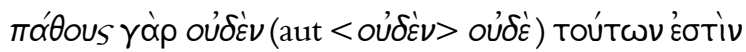
$\varepsilon^{\prime \prime} \xi \omega$

${ }^{41}$ Cf. Mestre et Gómez, op. cit., p. 171. 
Coray avoue que oúḋ̀v est une variante qu'il a trouvée dans les

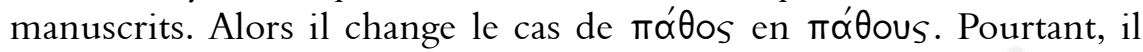
considère que la meilleure leçon qui émerge de ces deux variantes est

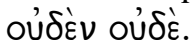

51. Pseudol. 6 (III.136.9) (p. 523)

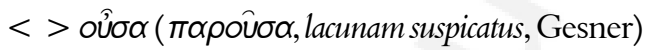

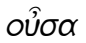

Coray réfute les corrections des philologues qui interviennent

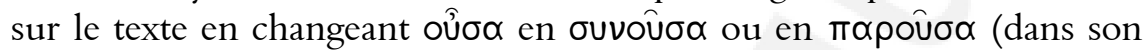

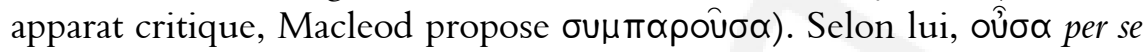
aliquando significat idem quod $\pi \alpha \rho \circ \hat{\sigma} \sigma$, quo sensu et hic accipiendum procul dubio est et il donne deux passages de l'Electre de Sophocle afin de

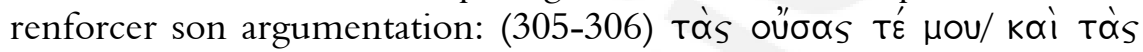

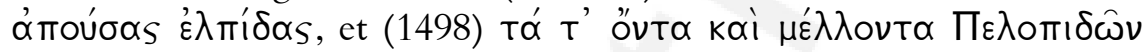

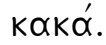

52. Pseudol. 16 (III.140.9) (p. 524)

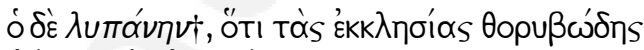

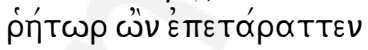

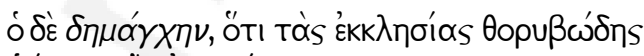

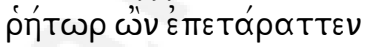

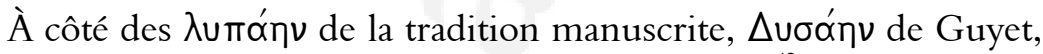
$\Delta u ́ \sigma \sigma \alpha v$ de Gesner, $\lambda u ́ \mu \eta v$ de Harmon et $\lambda u ́ m \eta v$ de Russo, ${ }^{42}$ Coray présente

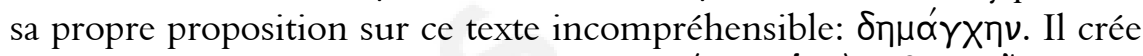

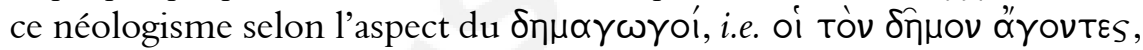

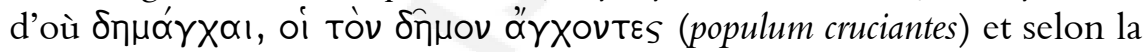
forme des ópoßá $\gamma X \eta$ (Galien 6.552 Kühn, Dioscoride 2.142) et v́á $\gamma X \eta$

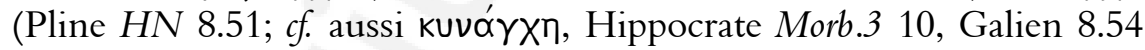

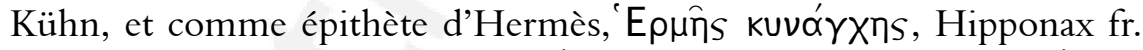

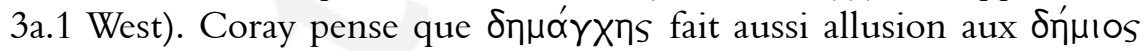
et $\delta$ nókolvos, "bourreau".
53. Pseudol. 20 (III.142.5)

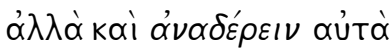
(p. 525)

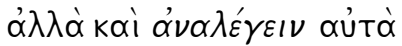

Coray considère que $\alpha^{\nu} v \delta^{\prime} \varepsilon \varepsilon \varepsilon^{\prime} \nu$ (detegere) n'est pas correct ici sémantiquement; l'infinitif doit se référer à quelque chose de différent. C'est pourquoi il propose $\alpha \dot{v} \alpha \lambda \lambda^{\prime} \gamma \varepsilon ı v$ (colligere undequa[que] et narrare).

\footnotetext{
${ }^{42}$ Cf. Russo, op. cit., p. 47-52: très bonne analyse du passage et de toutes les corrections proposées.
} 
54. Pseudol. 27 (III.145.7) (p. 528)

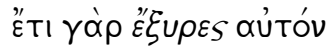

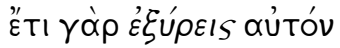

Coray a peut-être raison sur cette correction; il y a trois formes du verbe en question: $\xi u ́ p \omega, \xi u p \varepsilon ́ \omega$ et $\xi u p \alpha ́ \omega$. Lucien préfere surtout

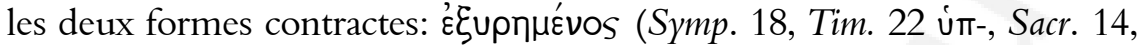

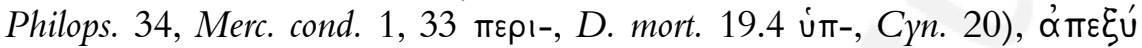

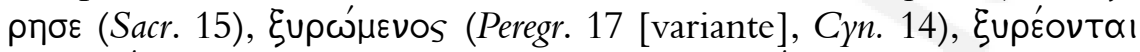

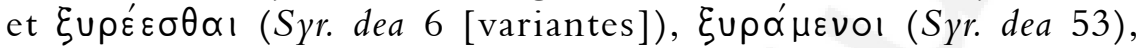

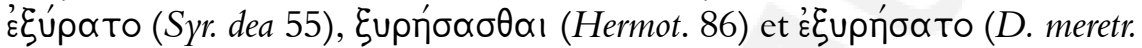

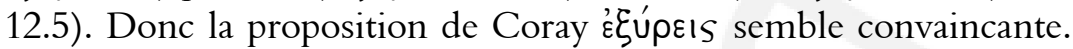

55. Tyr. 8 (III.161.20-21) (p. 485-486)

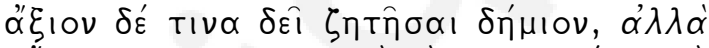

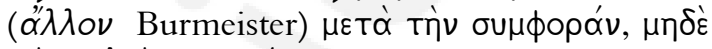

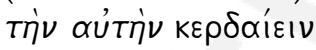

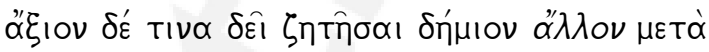

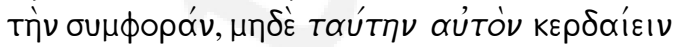

Coray interprète ainsi le passage corrigé: quarendus autem est alius quispiam carnifex illo dignius post [filii] casum; neque hunc illum lucrifacere oportet, i.e. neque patrem lucrifacere oportet filii casum.

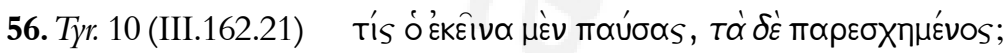

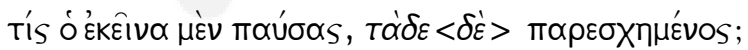

Aucune explication pour cette correction de la part de Coray. Le texte qu'il cite contient $\tau \alpha \alpha \delta \varepsilon$, donc il écrit $\tau \alpha{ }^{\prime} \delta \varepsilon \delta \varepsilon$ par analogie avec

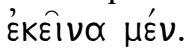

57. Fug. 27 (III.217.8) (p. 539)

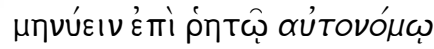

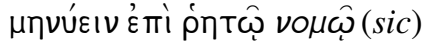

Coray corrige et interprète ainsi: certa ac definita quadam lege ut loquitur Cicero (Orat. 198), seu conditione rata atque constituta. Il est évident que $v o \mu \omega \hat{~ e s t ~ u n e ~ f a u t e ~ d ' o r t h o g r a p h e ~ d e ~ s a ~ p a r t ~ e t ~ q u ' i l ~ v o u l a i t ~ d i r e ~}$

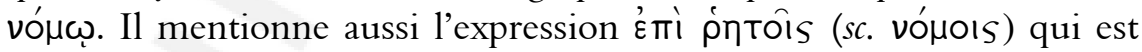
très connue dans la littérature grecque (e.g. Hérodote 5.57, Thucydide 1.122, Euripide Hipp. 459, et même Lucien Sat. 2).

58. Tox. 10 (III.229.11) (p. 501)

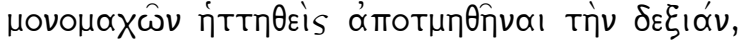

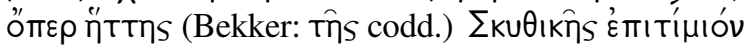
घंठTIV

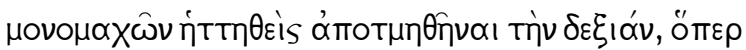

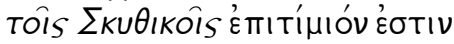


La correction que propose Coray ici nous paraît meilleure que celle de Bekker bien qu'il ne donne pas beaucoup d'explications: lego

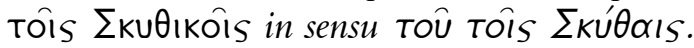

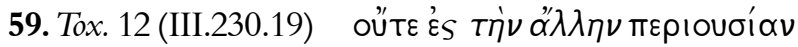

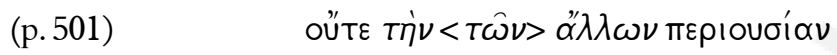

Coray dit que par ce passage existe aussi la variante $\tau \hat{\omega} \nu \stackrel{\alpha}{\lambda} \lambda \lambda \omega \nu$. Par conséquent, encore une fois, il considère que les deux variantes fournissent la vraie leçon tìv $\tau \hat{\omega} \nu \stackrel{\alpha}{\lambda} \lambda \lambda \omega \nu$.

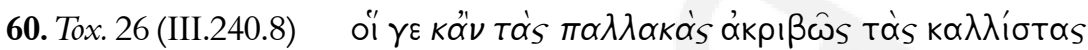
(p. 501)

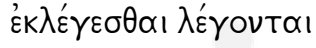

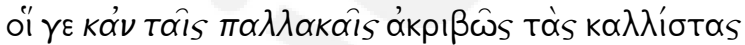

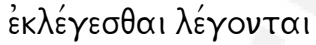

Coray considère que kåi $\alpha \dot{v}$ ici est une faute. Alors il corrige ou

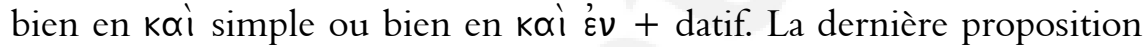
nous semble meilleure.

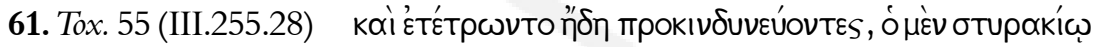
(p. 502)

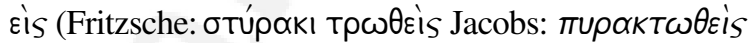
codd.) Tòv unpóv

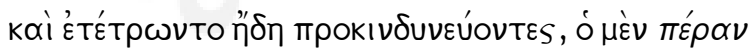

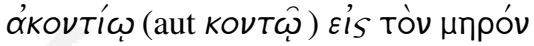

Coray propose sa correction en renvoyant le lecteur au $\$ 61 \mathrm{du}$

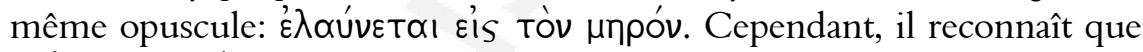
le "̌x $\chi \omega \nu$ mupíkautov de Asin. 6 lui indique de modifier son jugement sur cela. ${ }^{43}$

62. Dem. enc. 5 (III.263.27) (p. 545)

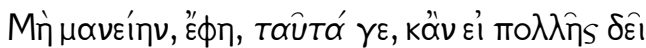

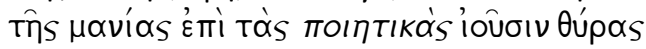

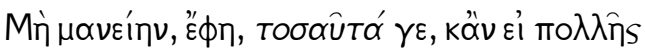

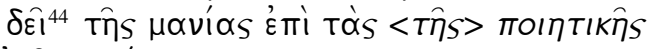
'oûoıv túpas

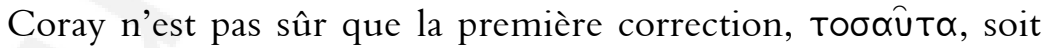
nécessaire car le mo $\lambda \lambda \hat{n} s$ qui suit lui paraît suffisant. Quant à la

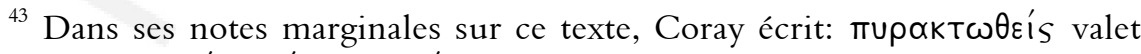

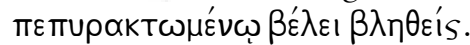

${ }^{44}$ Dans ses notes marginales sur ce texte, Coray propose $\delta^{\prime} \varepsilon_{0}$.
} 


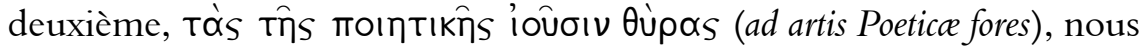
reconaissons qu'il a tort puisque le passage entier est une allusion à

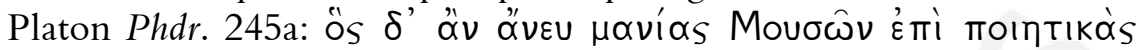

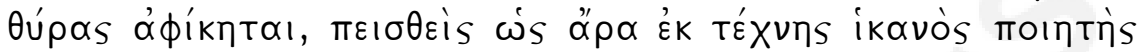

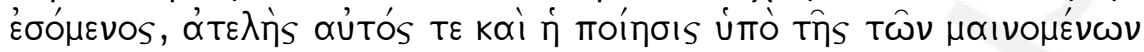

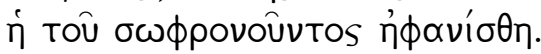

63. Dem. enc. 10 (III.266.5) (p. 547)

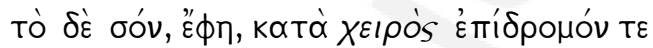

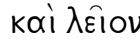

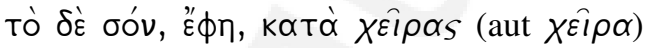

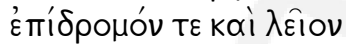

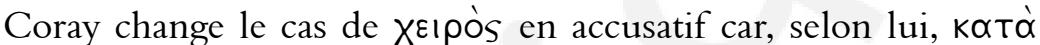

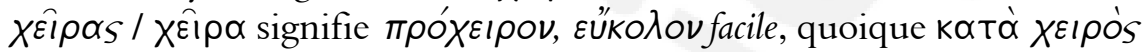
plane aliud significat; il renvoie encore une fois à Hésychios K1513: Kaтর̀

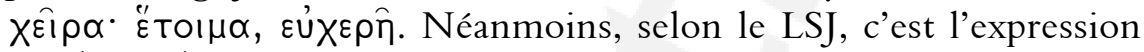

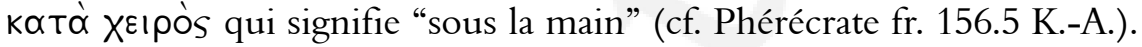

64. Dem.enc. 16 (III.269.5-6) (p. 548)

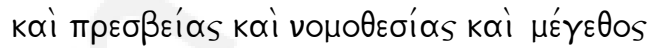

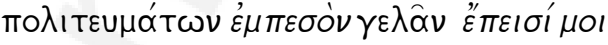

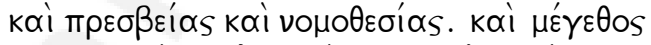

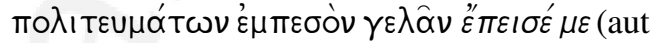

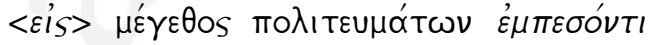

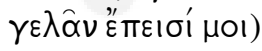

Coray trouve la syntaxe du passage fautive. D'abord il met un

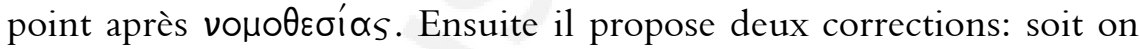

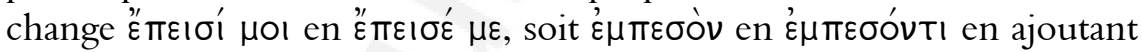

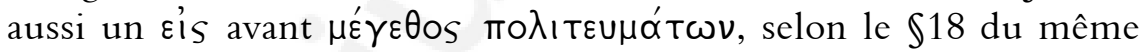

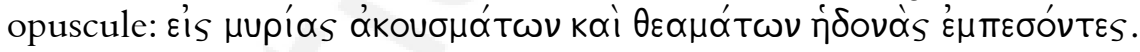

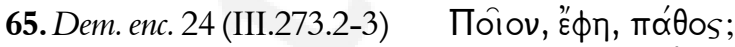
(p. 551)

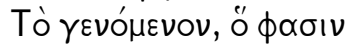

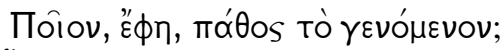

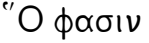

Une transposition dans ce dialogue lui donne plus d'élégance et éclaire le sens, admet Coray.

66. Dem.enc. 33 (III.278.3) (p. 552)

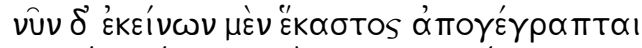

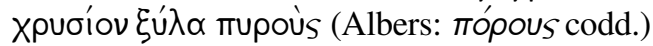

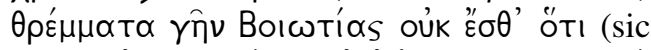

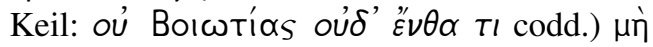
$\pi \alpha \rho '$ ' $\mu$ ноû $\lambda \alpha \beta o ́ v t \varepsilon s$ 


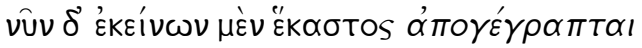

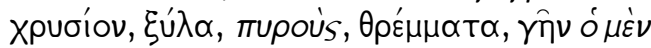

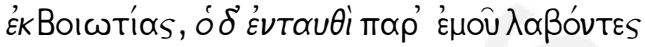

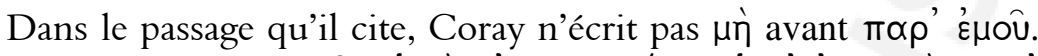

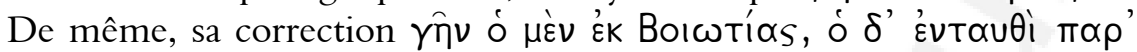

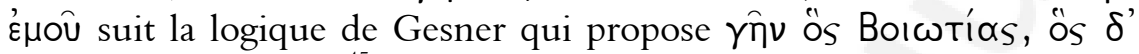

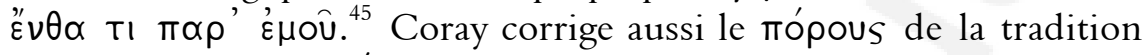
manuscrite en mupoús en renvoyant - comme le fait Macleod - à Demosthène 19.145.

67. Hist. co. 3 (III.288.14-15) (p. 479)

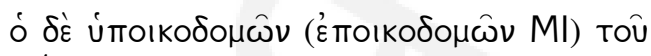
TÉ́XOUS

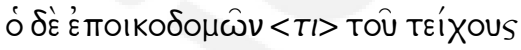

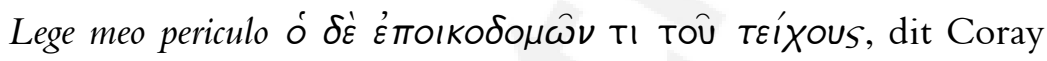
sans plus d'explications.

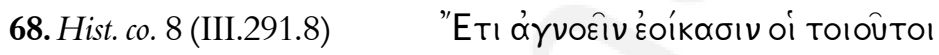
(p. 479)

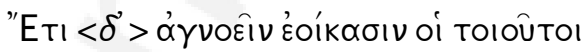
plus.

De même, aucune explication pour cette correction de style non

69. Hist. co. 51 (III.315.12) (p. 480)

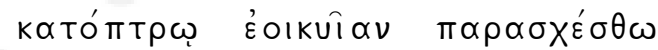

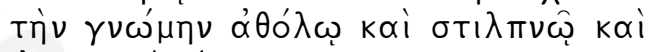

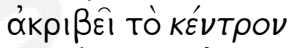

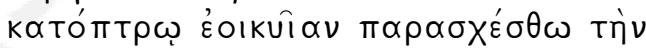

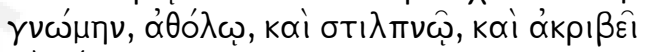
Tò KÉtpov

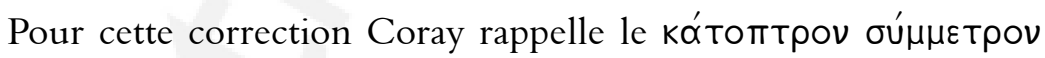
de $A d v$. ind. 29 et traduit par speculum justa magnitudinis.

70. Hist. co. 51 (III.315.14-15) ớ Yà (p. 480-482)

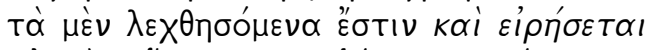

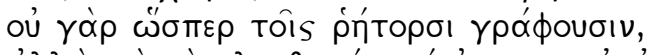

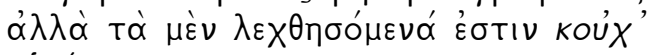

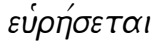

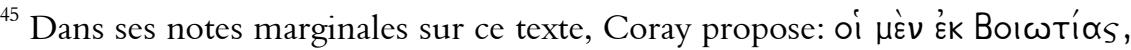
oi $\delta$ है $้ v \theta \varepsilon v$.
} 
Coray pense d'abord que les philologues ont du mal à comprendre

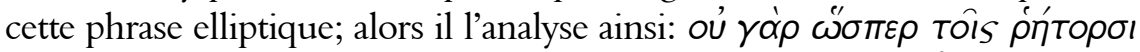

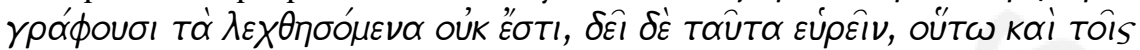

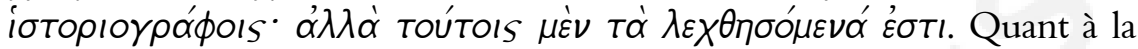

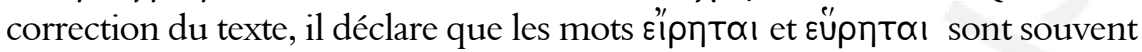
confondus par les copistes. Par conséquent, il corrige en '̇ं Tı koủX',

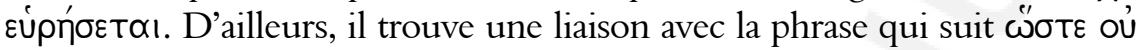

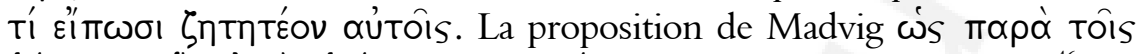

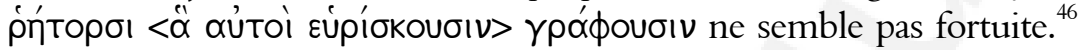

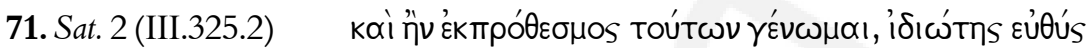
(p. 540)

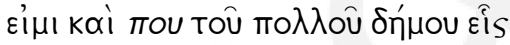

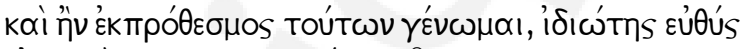

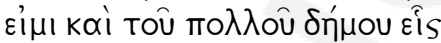

Aucune explication pour cette correction de la part de Coray.

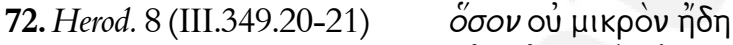

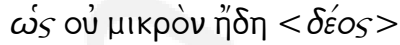

Pour cette correction Coray renvoie à $J u d$. voc. 2: $\delta^{\prime} \varepsilon_{0} \delta^{\prime} \delta^{\prime}$ oủ $\mu$ เкрóv

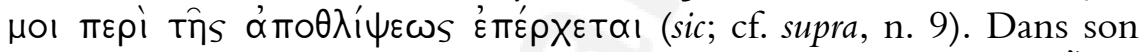
apparat critique, Macleod est d'accord avec Pelletus qui propose őбov

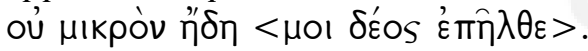

73.Zeux. 9 (III.355.15-16) (p. 478)

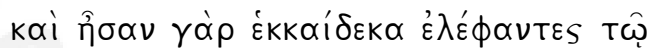

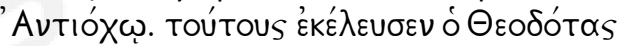

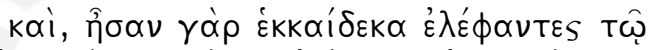

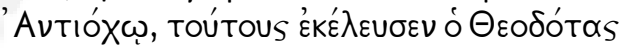

Coray fait une correction de ponctuation selon le style d'Hérodote.

74. Hermot. 17 (IV.31.7) (p. 473)

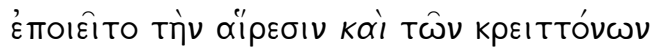

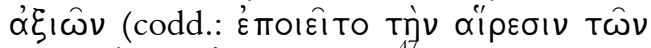

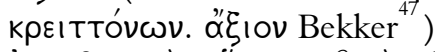

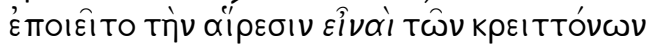

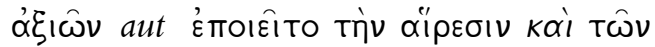

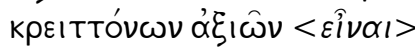

\footnotetext{
${ }^{46}$ Nesselrath (op. cit., 1984, p. 607) propose: oủ yà

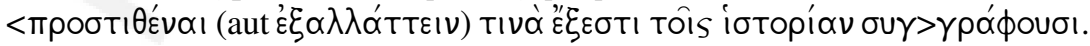

${ }^{47}$ Cf. Nesselrath, c.r. de Macleod, M. D. Luciani Opera. Vol. IV. Oxford: Oxford University Press, 1987 (Gnomon, Munich/Berlin, vol. 62, p. 504, 1990).
} 
Coray propose sa propre correction sur ce locus curruptus: soit il substitue Êıvaı à kớ, soit il ajoute Êıvaı à la fin. Dans l'édition du texte la plus récente, Möllendorff supprime seulement le kai. ${ }^{48}$

75. Hermot. 21 (IV.34.7) (p. 474) ¡̋

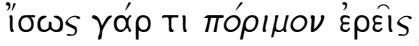

Coray change $\gamma \nu \omega ́$ pınov - que la tradition manuscrite n'a jamais

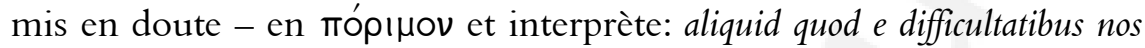
expediat. Il renvoie le lecteur aux paragraphes $26-27$ où le mot ámopía

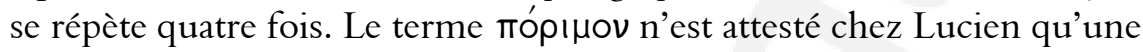
seule fois, dans Dem. enc. 14.

76. Hermot. 32 (IV.42.15) (p. 474)

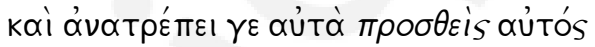

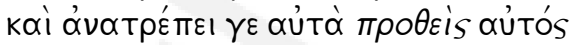

De même, Corray corrige ici $\pi \rho \circ \sigma \theta \varepsilon i s$ en $\pi \rho \circ \theta \varepsilon i s$ car quelques lignes plus bas, dans le $\$ 33$, le participe $\pi \rho \circ \theta_{\varepsilon}^{\prime} \mu \varepsilon v o$ os est répété trois fois.

77. Hermot. 52 (IV.59.10) (p. 475)

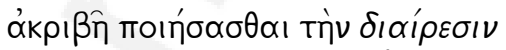

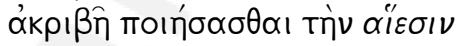

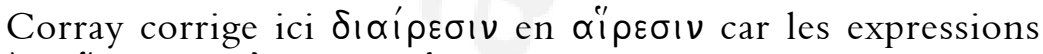

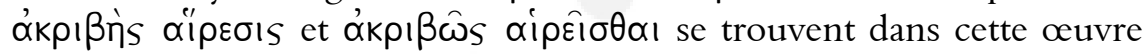
cinq fois: paragraphes $21,54,56,64$ et 67.

78. Hermot. 55 (IV.61.11) (p. 475)

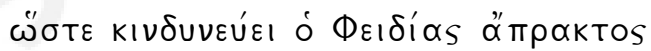

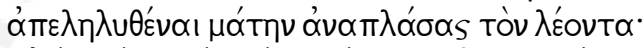

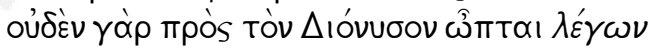

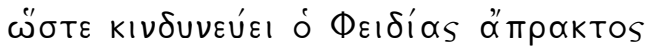

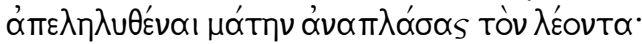

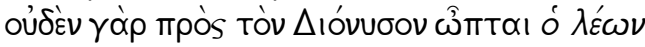

Coray mentionne que quelques philologues corrigent $\omega \hat{\pi} \pi \propto$ ।

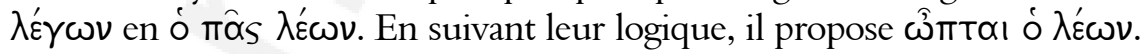

79. Hermot. 74 (IV.76.19) (p. 476)

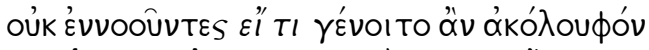

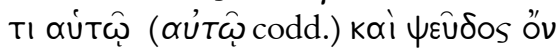

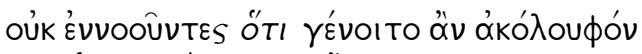

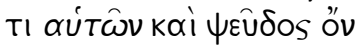

${ }^{48}$ Cf. von Möllendorff, op. cit. 
Coray corrige le texte ainsi et explique: non advertentes animum, quod consequens aliquid eorum fieri (vel videri) potest, quamvis falsum sit.

80. Prom. verb. 3 (IV.86.26)

(p. 455)

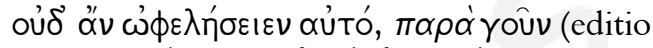

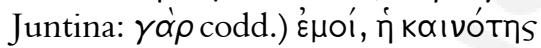

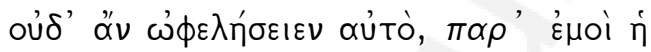
kaıvótms

Il s'agit de la première correction de Coray sur le texte de Lucien. Pourtant, il n'en donne aucune explication.

81. Nav. 20 (IV.107.8)

(p. 532)

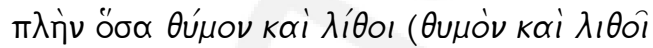

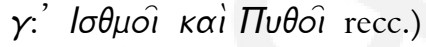

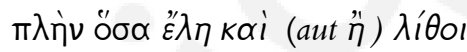

Coray admet que sa correction est une reformulation des propositions d'autres philologues.

82. Nav. 22 (IV.108.16)

(p. 533)

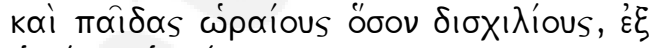

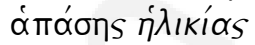

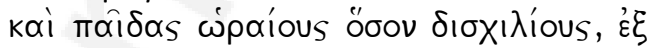

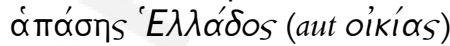

Coray trouve qu'ici il y a une contradiction entre $\pi \alpha \hat{\imath} \delta \alpha_{S}$ et $\dot{\varepsilon} \xi$

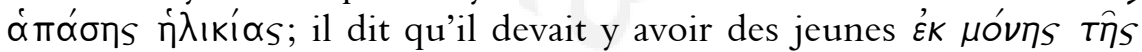

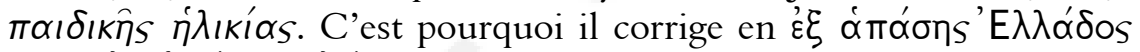

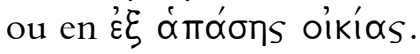

83. Nav. 32 (IV.113.10) (p. 533) $\delta ı \alpha \lambda \alpha^{\prime} \nu \omega \mu \varepsilon \nu \quad\left(\delta ı \alpha \beta \alpha^{\prime} \lambda \omega \mu \varepsilon \nu\right.$ Gesner:

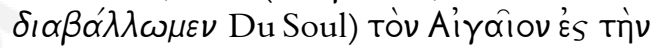

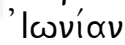

$\delta ı \alpha \beta \alpha i v \omega \mu \varepsilon v$ (aut $\delta ı \alpha \beta \omega \hat{\mu} \mu \varepsilon v$ ) tòv Airôiov

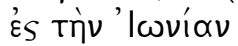

Coray corrige ainsi le texte en renvoyant le lecteur quelques lignes

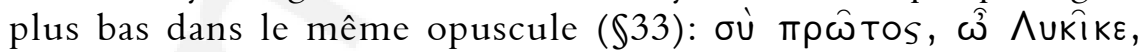

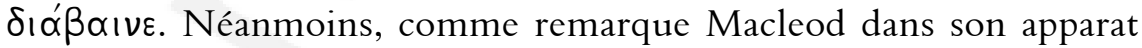
critique, la proposition de Gesner fait allusion à Thucydide 6.30: Tòv

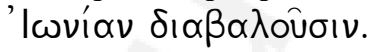

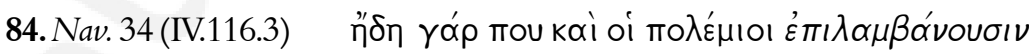

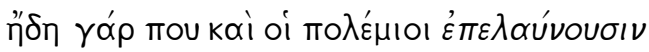

Pour cette correction, Coray se base sur la quadruple répétition des

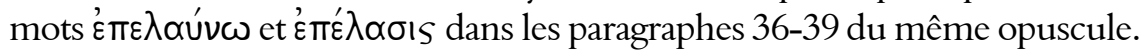


85. Cyn. 1 (IV.134.2)

(p. 553-554)

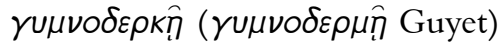

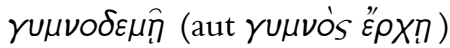

Coray trouve que le mot $\gamma u \mu v o \delta \varepsilon \mu \eta ़$ est plus proche du sens de

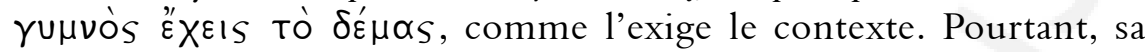
deuxième proposition nous paraît meilleure, et comme le pense Coray

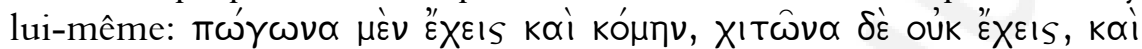

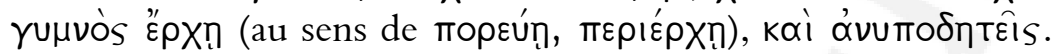

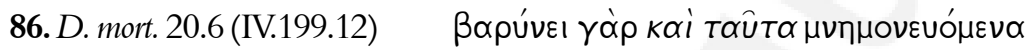
(p. 462)

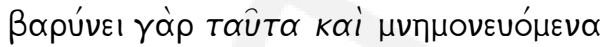

Aucune explication pour cette correction de la part de Coray. Dans son apparat critique, Macleod se demande: an $\beta \alpha \rho u v \varepsilon \hat{\imath}$ ?

87. D. meretr. 4.5 (IV.326.9)

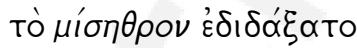
(p. 536)

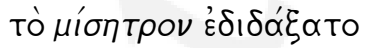

Coray pense que le mot $\mu$ íontpov est formé par analogie à

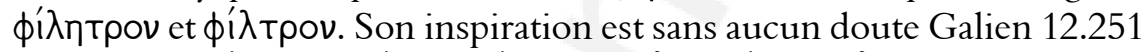

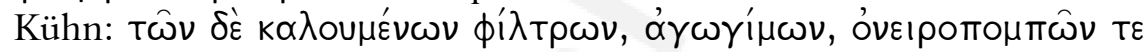

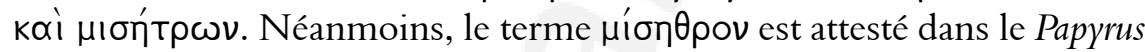

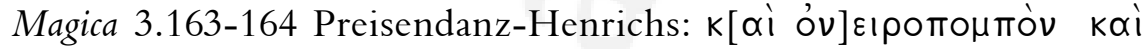

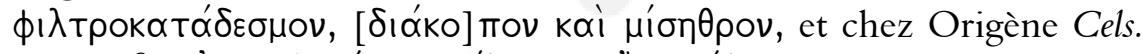

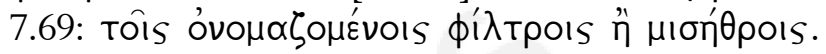

88. D. meretr. 9.3 (IV.341.16) (p. 537)

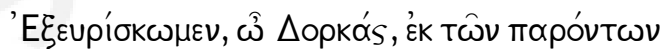

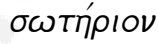

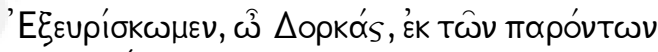

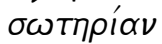

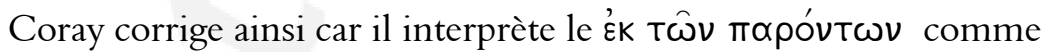

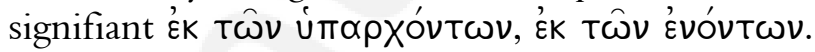

89. D. meretr. 9.4 (IV.342.10) (p. 537)

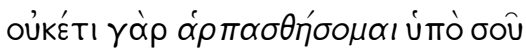

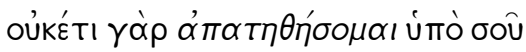

Correction pertinente tout à fait conforme au contexte de l'opuscule. Cependant, Coray n'en donne pas d'explication.

90. D. meretr. 11.1 (IV.348.8)

(p. 538)

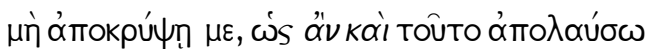

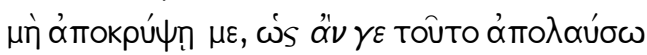

Encore une correction de style sans explications de la part de Coray. 
91. Philopatr. 1 (IV.367.10) (p. 556)

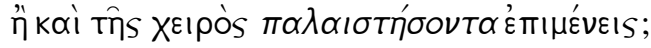

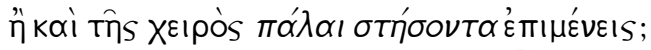

Coray corrige ainsi et traduit en français: "ou attends-tu déjà qu'on t'arrête en te prenant par la main?".

92. Philopatr. 3 (IV.370.2) (p. 556)

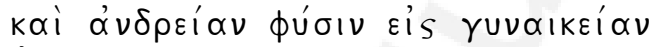

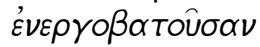

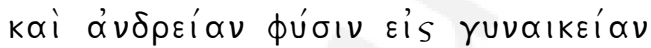
$\mu \varepsilon \tau \varepsilon \rho \chi \circ \beta \alpha \tau o \hat{\sigma} \sigma \alpha \nu$

Coray crée ce néologisme par la combinaison des verbes

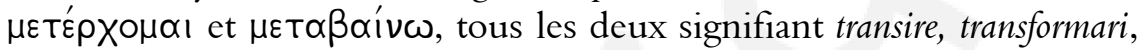
puisqu'ici il s'agit de la transformation d'un homme en une femme. C'est pour cette même raison que nous trouvons plus intelligente la

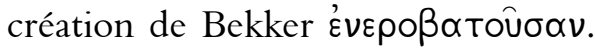

93. Philopatr. 9 (IV.374.17)

(p. 557)

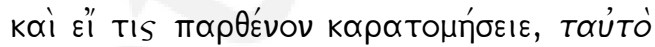

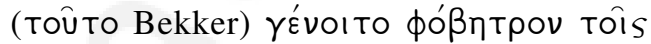

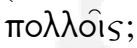

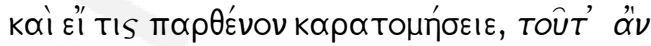

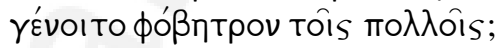

Coray ne donne pas d'explication pour la correction qu'il fait. Il suit quand même la logique de Bekker.

94. Philopatr. 20 (IV.383.3)

(p. 557-559)

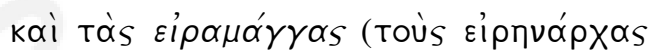
Gesner: тoùs $\alpha \lambda \lambda \eta \lambda \varepsilon \gamma \gamma$ úos Baldwin) $\delta^{\prime} \varepsilon \dot{\xi} \varepsilon \tau \alpha$ ।

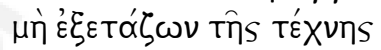

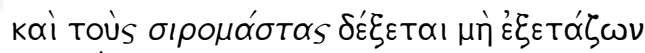

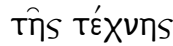

Coray fait une très bonne correction dans ce texte corrompu qui

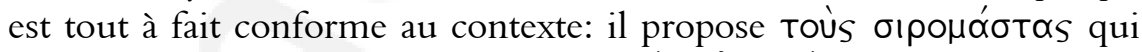

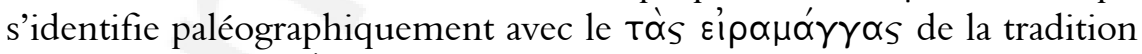

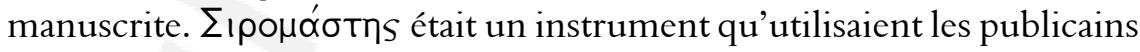
dans leurs recherches (Eunape Hist. 1.245; cf. aussi Et. Gud. s.v. бєıроно́⿴囗тा) ). Coray pense ici qu'il s'agit d'une métonymie et que le mot en question veut dire les publicains, ce qui est logique puisque le passage parle des créanciers et des débiteurs. Il l'interprète ainsi: Publicanos etiam admittet, parum sollicitus de arte quam profitentur [dummodo ad bonam frugem se recuper(ar)e promittant]. 
95. Philopatr. 21 (IV.383.17) (p. 560)

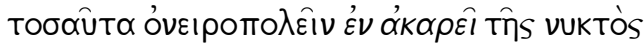
oüons

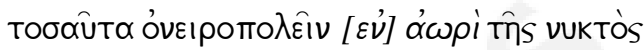
oüons

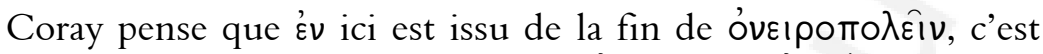

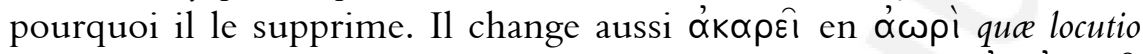

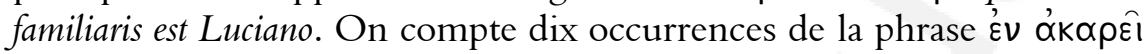
chez Lucien en regard de cinq de ơ $\omega$ i. Cependant Philopatris est une œuvre apocryphe.

96. Philopatr. 22 (IV.384.8)

(p. 560)

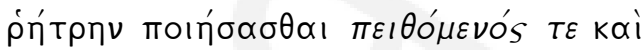

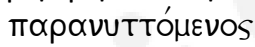

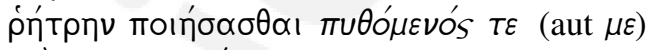

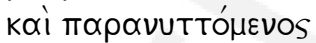

Cette correction de Coray porte surtout sur l'ajustement chronologique entre l'infinitif aoriste поı́ंб $\sigma \theta \alpha ı$ et le participe présent

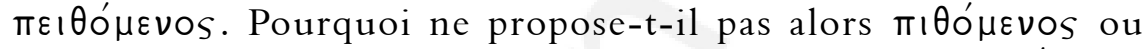

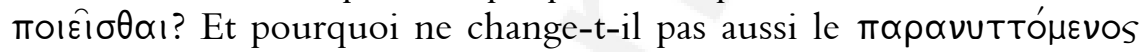
qui suit?

97. Charid. 17 (IV.398.12) (p. 568)

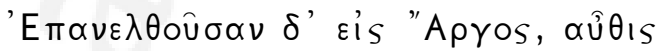

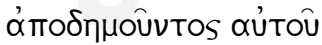

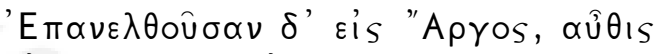

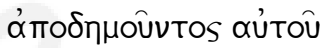

Coray corrige ainsi la ponctuation du texte car $\alpha \hat{\theta} \theta ı s$ non ad

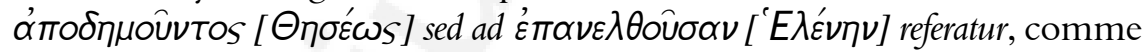
il le dit.

On vient de présenter quatre-vingt-dix-huit passages de Lucien corrigés par Adamantios Coray, représentant un septième des Animadversiones in Lucianum. Beaucoup d'entre eux méritent de trouver leur place non seulement dans l'apparat critique des éditions lucianesques mais dans le texte même. Un assez grand nombre de ces corrections ont été vérifiées par la tradition manuscrite ou ont été proposées postérieurement par d'autres philologues. ${ }^{49}$ Quelques-unes paraissent hyperboliques, superflues, non convaincantes. Pourtant, il y en a d'autres qui semblent naturelles comme si elles avaient été écrites par Lucien luimême. On pourrait citer comme exemples les propositions faites à propos

\footnotetext{
${ }^{49}$ Cf. Kalospyros, op. cit., p. 87-105 (vol. II).
} 
de Cat. 19 [corr. n ${ }^{\circ}$ 5] et $A \sin .28$ [30]: la simplicité de la correction effectuée est stupéfiante, et il est même étonnant que personne n'ait songé plut tôt à quelque chose qui paraît aussi évident. On est également surpris par les observations stylistiques de Coray (e.g. Zeux. 9 [73], Charid. 17 [97]) et par son instinct philologique admirable (J. trag. 44 [6], Luct. 16 [35], Tox. 26 [60]). Coray n'hésite pas à créer des néologismes (Pseudol. 16 [52], Cyn. 1 [85], Philopatr. 3 [92]) ou à résoudre des problèmes textuels qui semblaient insolubles jusque là (Icar. 20 [9], Syr. dea 60 [42], Philopatr. 20 [94]). On est surpris par la richesse de ses connaissances sur la littérature et la langue grecques, chaque fois qu'il cite des passages parallèles d'autres auteurs (Paras. 42 [17], Alex. 48 [39], Lex. 6 [43], Pseudol. 6 [51]) ou de Lucien même (Hist. co. 51 [69], Hermot. 21 [75], 32 [76], 52 [77], Nav. 34 [84]) et des lemmes d'ancients lexicographes (Merc. cond. 26 [25], Asin. 40 [32]).

Coray savait lire avec précision, comprendre en profondeur et interpréter correctement les textes. Dans l'une de ses lettres à Chardon de la Rochette (2/9/1793) il a écrit qu'il pensait publier ses Observationes Miscellanex: "mes Mélanges, quoique j'y compte maintenant près de six cents pages d'écriture, sans les remarques sur Hérodote et Athénée, qui sont écrites dans des cahiers séparés, ne sont pas encore suffisants pour former un petit volume in $8^{\circ}$; car je compte n'y mettre absolument que les corrections qui ont un haut degré de probabilité, et en écarter

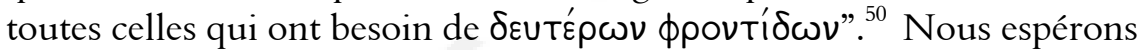
avoir pu rendre réelle au moins une part de sa vision aujourd'hui.

\section{Appendice: le ms. Chios 379 et les marginalia}

Le ms. Chios 379 est composé de six pages qui contiennent soixante-huit corrections d'Adamantios Coray sur le texte lucianesque des volumes II à VIII de la Bipontine. ${ }^{51}$ Elles sont écrites en deux colonnes et comportent moins d'explications que dans le ms. 490. On en cite ici trente-trois qui n'ont été proposées par aucun philologue jusqu'à présent.

\footnotetext{
${ }^{50}$ Cf. Kalospyros, op. cit., p. 473 (vol. I).

${ }^{51}$ Le Professeur G. A. Christodoulou pense qu'il devait y avoir d'autres pages qui contenaient les corrections des volumes I (Somn., Prom. verb., Nigr., Jud. voc., Tim., Alc., Prom.) et IX (Sat., Symp., Syr. dea, Dem. enc., Deor. conc., Cyn., Sol., Philopatr., Ner.). Je voudrais le remercier pour m'en avoir fourni des photocopies. Le ms 379 est aussi mentionné par Êalospyros, op. cit., p. 218 (vol. I, n. 58).
} 
98. Hipp. 6(I.14.22)

99. Nigr. 37 (I.44.28)

100. Nigr. 38 (I.45.21)

101. Macr. 4 (I.74.7)

102. V.H.II 9 (I.107.5)

103. Cat. 11 (I.187.12)

104. Prom. 5 (I.280.8)

105. Tim. 6(I.312.10-11)

106. Tim. 11 (I.314.19)

107. Pisc. 47 (II.80.10)

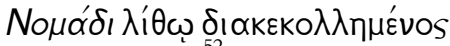
1.[ege] $\lambda \circ \gamma \alpha \alpha^{\prime} \delta_{1}^{52}$

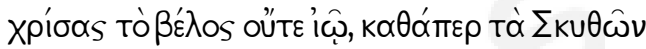

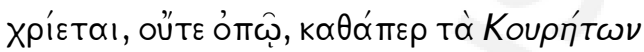

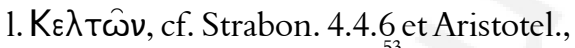
Mirabilium auscultationes $837 \mathrm{a}^{53}$

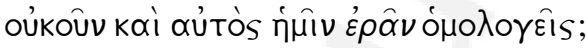
f.[orsan] бuvepồ

Bóktpols

f. Baktpíols

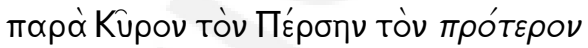

f. $\pi \rho \varepsilon \sigma \beta u ́ t \varepsilon \rho \circ \nu$

Ei ốóv Te Eîvaı

1. กิ่

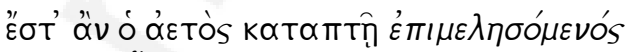

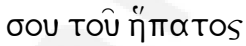

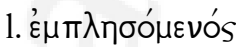

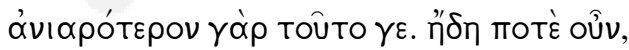

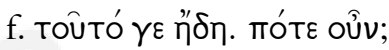

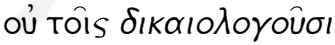

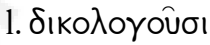

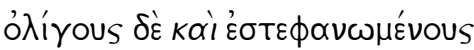

dele kai

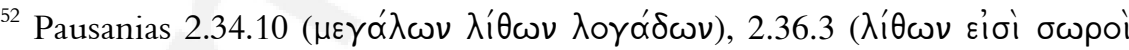

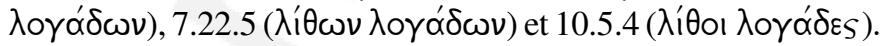

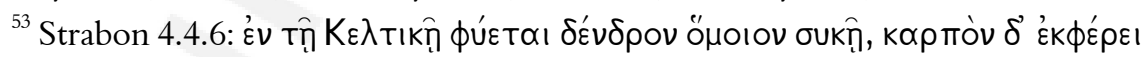

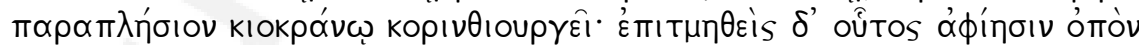

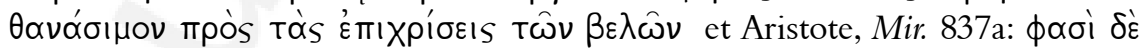

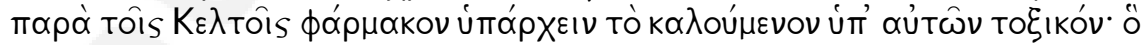

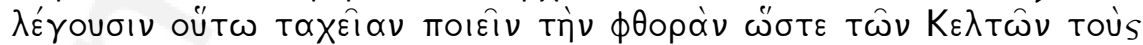

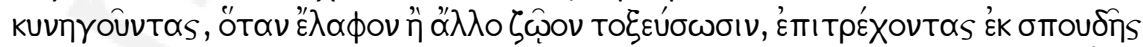

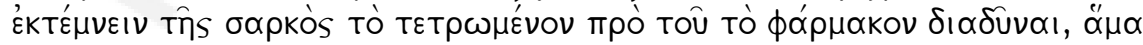

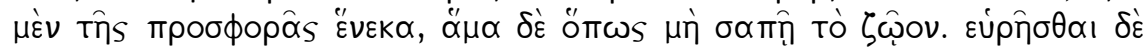

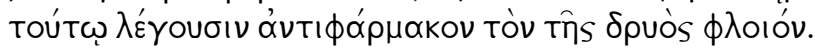




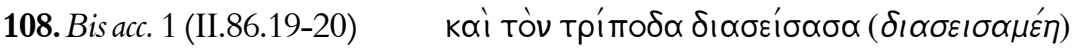

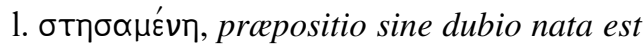
ex ultima тоû трі́ тоб $\alpha$

109. Bis acc. 1 (II.87.7)

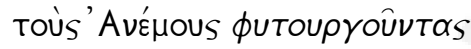
1. UंтоupYoûvTas

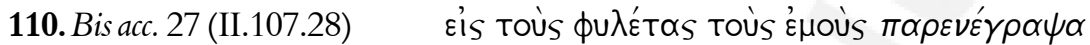

111. Paras. 2 (II.145.9)

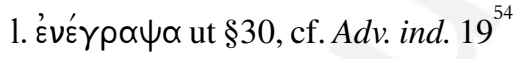

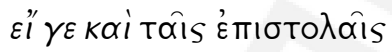

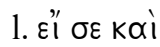

112. Paras. 3 (II.146.4-5)

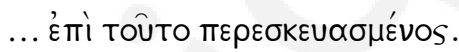

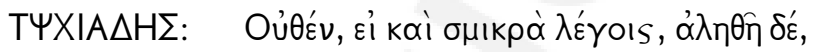

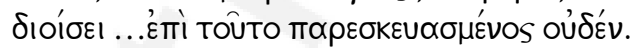

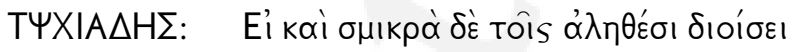

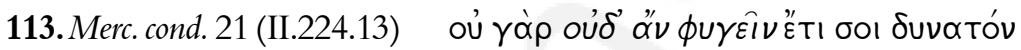

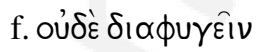

114. $A \sin .6($ II.279.18)

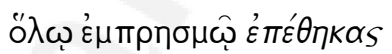

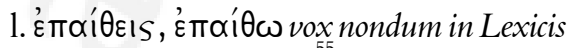

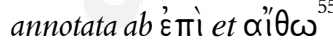

115. $A \sin .37$ (II.297.4)

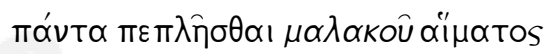

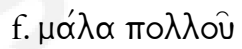

116. Asin. 48 (II.303.33)

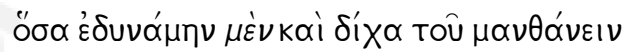
dele $\mu \grave{\varepsilon v}$

117. Alex. 5 (II.334.2)

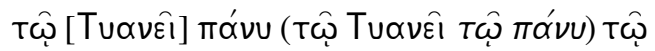

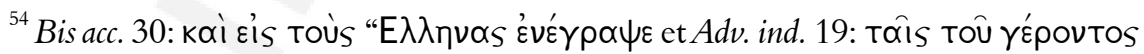

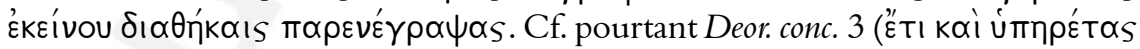

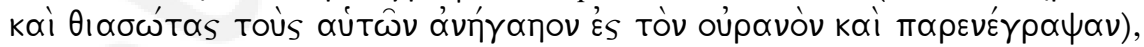

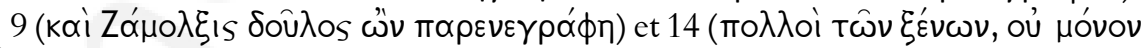

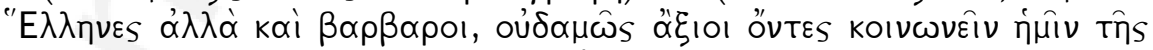

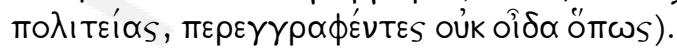

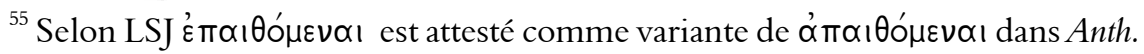
Gr. 7.48.2.
} 
118. Alex. 6 (II.334.8)

119. Alex. 10 (II.336.1)

120. Salt. 26 (III.36.19)

121. Amor. 1 (III.85.10)

122. Amor. 6 (III.88.20-21)

123. Amor. 10 (III.90.9)

124. Pseudol. 16 (III.140.12)

125. Tox. 46 (III.250.15)

126. Tox. 57 (III.257.7-8)

127. Tox. 61 (III.259.10)

128. D. mort. 15.2 (IV.185.18)

129. D. mort. 29.2 (IV.227.18)

130. D. deor. 2.4 (IV.265.8)
Távu non exstat in cod. $\beta$, erratum esse ex verbis T⿳亠丷

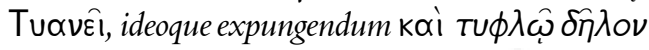

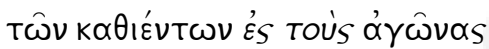
1. ÉaUTOUंS ÉSTOUंS

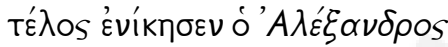

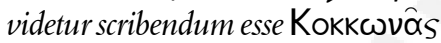

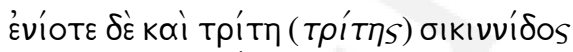

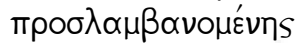

1. трі́тп тทิs

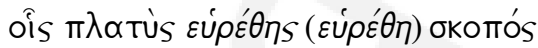

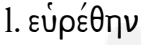

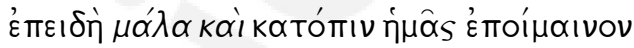
aûpa।

1. $\mu \alpha \lambda \alpha \kappa \alpha i$

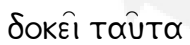

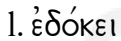

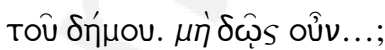

1. oủ $\mu$ मे

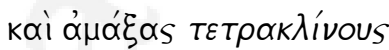

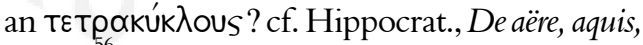
locis $18^{56}$

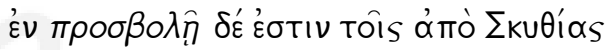

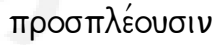

1. $\pi \rho \circ \beta \circ \lambda \bar{n}$

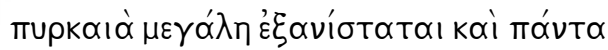
$\pi \varepsilon \rho І \varepsilon \kappa \lambda \varepsilon i \varepsilon т 0$

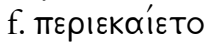

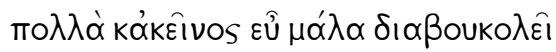

1. $\dot{\alpha} \lambda \lambda \dot{\alpha}$

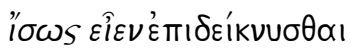

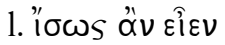

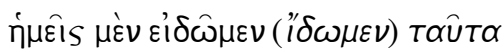

1. $\delta i \delta \circ \mu \varepsilon v$ vel $\delta ı \delta \omega \hat{\mu} \varepsilon v$

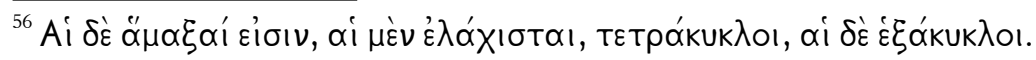


Les notes marginales d'Adamantios Coray sur le texte de Lucien s'étendent tout au long des neuf premiers volumes de l'édition Bipontine. Malheureusement le deuxième volume de la collection qui contient les Dialogues des dieux, le Jugement des déesses, les Dialogues marins et les Dialogues des morts est perdu. On a compté plus de quatre cents corrections: quelques-unes ont été copiées dans les mss. 379 et 490 (Coray a choisi les meilleures) et beaucoup d'entre elles ont été vérifiées par la tradition manuscrite ou ont coïncidé avec les corrections d'autres philologues. Néanmoins, on y trouve cent vingt-quatre propositions originales que nous présentons ci-dessous.

\begin{tabular}{|c|c|}
\hline 131. Phal.A4 (I.2.27) & 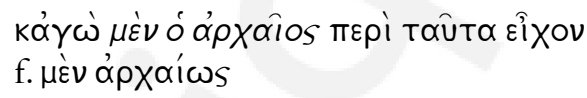 \\
\hline 132. Hipp. 8 (I.15.6) & 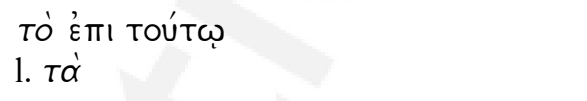 \\
\hline 133. Bacch. 4 (I.18.1) & $\begin{array}{l}\dot{\varepsilon} \alpha \lambda \omega^{\prime} \kappa \varepsilon \sigma \alpha \nu \\
\text { 1. } \dot{\varepsilon} \alpha \dot{\alpha} \lambda \sigma \alpha \nu \nu\end{array}$ \\
\hline 134. Herc. 4 (I.21.20) & 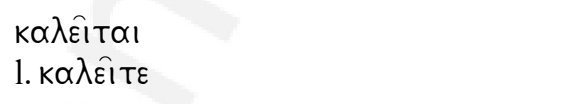 \\
\hline 135. Nigr. 6 (I.33.23) & 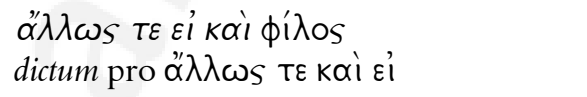 \\
\hline 136. Nigr. 34 (I.43.24) & 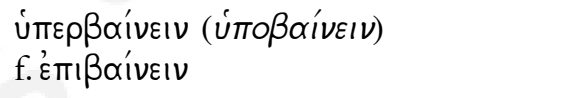 \\
\hline 137.Demon. 10 (I.48.18) & 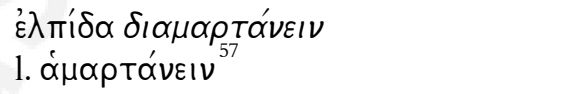 \\
\hline
\end{tabular}

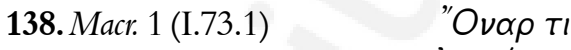

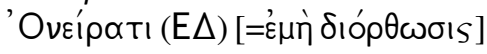

139. V.H.II 34 (I.118.14)

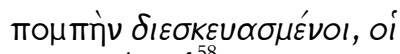

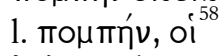

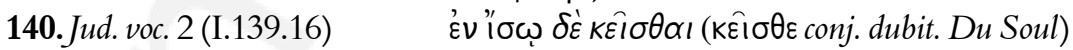

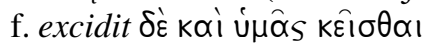

\footnotetext{
${ }^{57}$ Il s'agit d'une correction habituelle de la part de Coray: il pense que la préposition $\delta ı \alpha$ - est issue de la dernière syllabe $-\delta \alpha$ du mot précédent.

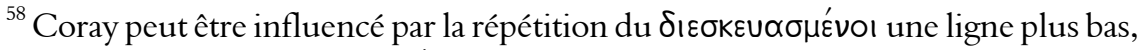

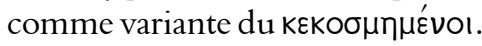


141.Jud. voc. 3 (I.140.4)

142. Sol. 7 (I.170.7)

143. Cat. 2 (I.179.6)

144. Cat. 11 (I.187.6)

145. Cat. 22 (I.195.9)

146. J. conf. 6 (I.205.14)

147.J. trag. 51 (I.247.5)

148. Gall. 8 (I.256.3)

149. Gall. 20 (I.266.18)

150. Gall. 24 (I.270.16)

151. Icar. 3 (I.291.7)

152. Vit. auct. 14 (II.36.5)

153. Pisc. 42 (II.77.7)

154. Adv. ind. 18 (II.129.1)

155. Adv. ind. 20 (II.130.1)

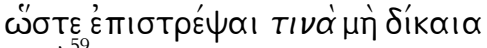
f. $\operatorname{ta}^{59}$

$\pi \hat{s} \alpha^{\alpha} \nu T I \pi 010 u ́ \mu \varepsilon v O S$

1. $\pi \omega \hat{s}$ oưk

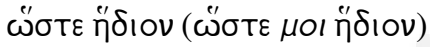

f. kai

É Tívos

f. Tívos åv

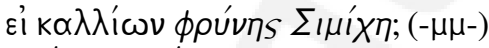

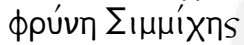

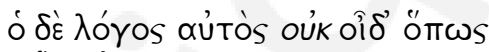

f. òv oưk

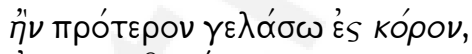

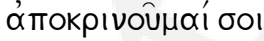

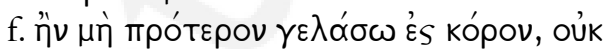

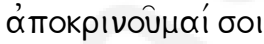

$\delta \alpha \psi \mid \lambda \dot{\varepsilon} S$ Tò $\delta \varepsilon \hat{\imath} \pi \nu \circ v$

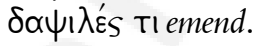

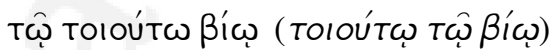

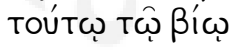

ध́kTòs ó $\mu \dot{\varepsilon} \nu$

غKTTòs $\mu \dot{v} \nu$

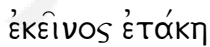

f. ó knpòs

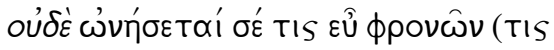
om. Bipontina)

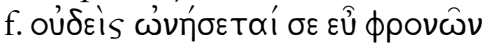

$\varepsilon \nu \beta \rho \alpha \chi \varepsilon \hat{\varepsilon}$

1.

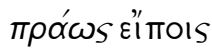

f. $\pi \rho \omega \hat{T}$ Tov $\ddot{\alpha} \nu$

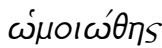

1. ópolos $\varepsilon \hat{\imath}$

${ }^{59}$ Dans ses Observationes Miscellanea, p. 456, Coray corrige en $\tau ı \nu<i ~ \tau>\alpha ́$, une proposition faite aussi par Castigliori [cf. l'apparat critique de Bompaire, op cit., p. 179 (vol. II)]. 
156. Adv. ind. 24 (II.132.1)

157. Adv. ind. 25 (II.132.13)

158. Somn. 3 (II.136.17)

159. Somn. 10 (II.139.3)

160. Somn. 15 (II.140.22)

161. Paras. 1 (II.142.3)

162. Paras. 8 (II.149.11)

163. Paras. 51 (II.169.9)

164. Paras. 53 (II.170.12)

165. Paras. 57 (II.172.9)

166. Philops. 8 (II.180.9)

167. Philops. 9 (II.181.10)

168. Philops. 9 (II.181.14)

169. Philops. 12 (II.182.18)

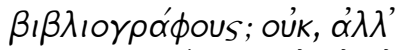

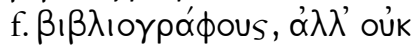

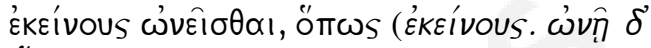
ö $\left.\mu \omega_{S}\right)$

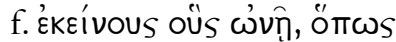

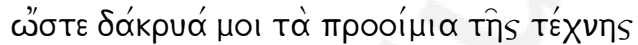
1. TÉXVחS ก่̂V. nam ellipsis hic non est ferenda

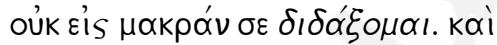

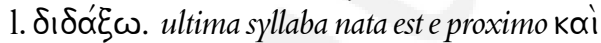

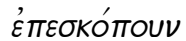

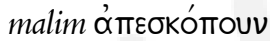

$\delta \delta^{\prime}$ oủ $\alpha^{\prime} v$ TI dele $\delta i$

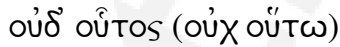

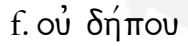

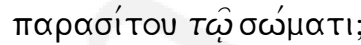

dele $\mathrm{T} \hat{\omega}$

пврі тоUंTous (ToútoIs)

1. $\pi \alpha \rho \grave{~}$

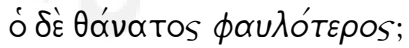

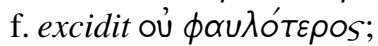

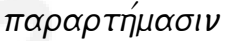

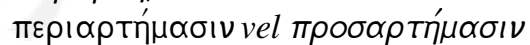

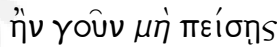

f. $\mu \eta^{\prime} \mu \varepsilon$

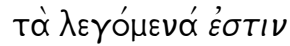

f. है०Tळ।

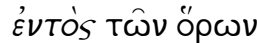

f. ह́кTòs

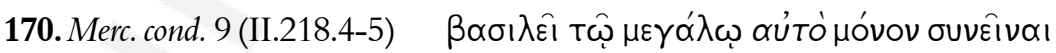

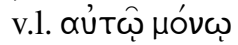

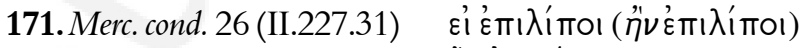
пे

172. Anach. 1 (II.237.8) kai भ̈vidoù

1. $v \hat{v} \nu$ 
173. Anach. 9 (II.239.16)

174. Asin. 4 (II.278.4)

175. Asin. 16 (II.284.28)

176. Asin. 27 (II.291.18)

177. Asin. 27 (II.291.22)

178. Asin. 40 (II.298.29)

179. Asin. 56 (II.309.5)

180. Alex. 9 (II.335.18)

181. Luct. 20 (II.315.23)

182. Rh.pr. 11 (II.323.4)

183. Rh.pr. 23 (II.328.17)

184. Syr. dea 11 (III.4.26)

185. Syr. dea 45 (III.21.6)

186. Salt. 29 (III.37.19)

187. Lex. 20 (III.66.11-12)

188. Astr. 29 (III.84.9)

189. Pro im. 29 (III.132.8) $\mu \varepsilon \gamma \alpha \lambda \circ \delta \omega \rho \varepsilon \hat{\alpha}$

1. $\mu \varepsilon \gamma \propto \lambda \circ \delta \omega \omega \operatorname{iq}^{60}$

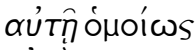

aưtriv

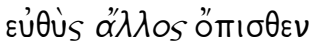

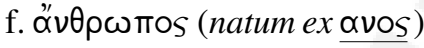

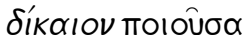

v.l. $\delta^{\prime} \mid k \alpha ı \alpha$

ws övov

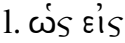

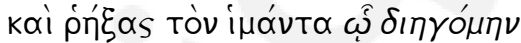

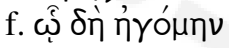

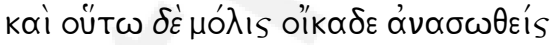

v.l. סं

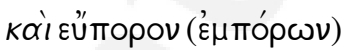

dele kai

oủk $\alpha ّ \nu ~ o i o ́ \mu \varepsilon \theta \alpha(o i \omega ́ \mu \varepsilon \theta \alpha)^{61}$

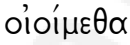

$\delta ı \alpha^{\prime} \xi \varepsilon \tau \alpha \_$

1. $\delta I \delta \alpha \xi^{\prime} \xi 1$

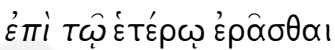

f. $\varepsilon$ ¿ाi $T \omega$

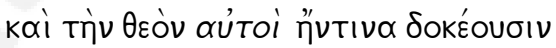

f. aủrinv

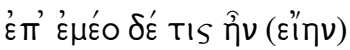

f. "̋ๆv

vevó

v.l. $v \varepsilon v \varepsilon \varepsilon \mu \eta \kappa \varepsilon v$

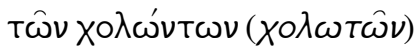

$x \circ \lambda \omega \delta \omega \nu$

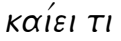

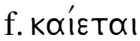

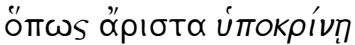

f. úTOKрIVEิ

\footnotetext{
${ }^{60}$ Cf. Sat. 4: $\mu \varepsilon \gamma \alpha \lambda \circ \delta \omega \rho i \alpha s$, et D. mort. $16.4 \mu \varepsilon \gamma \alpha \lambda \circ \delta \omega \rho \varepsilon \alpha \underline{\alpha}$ ( $\mu \varepsilon \gamma \alpha \lambda \circ \delta \omega \rho i ́ \alpha$ Bekker). ${ }^{61}$ Cf. Andò, op. cit., p. 63.
} 
190. Pseudol. 30 (III.147.6)

191. Peregr. 28 (III.198.18)

192. Peregr. 37 (III.202.2)

193. Peregr. 37 (III.202.3)

194. Fug. 33 (III.221.12)

195. Tox. 20 (III.236.11)

196. Tox. 34 (III.244.2-3)

197. Dem.enc. 1 (III.262.3)

198. Dem.enc. 10 (III.266.6-7)

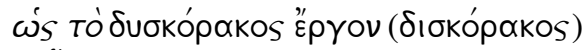
f. $\omega ॅ \sigma \tau$

VUKTÉpIOV

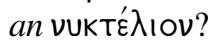

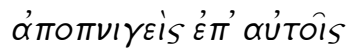

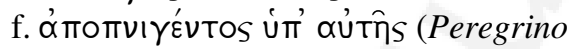
sc. suffocato ủmò Tท̂s mupâs)

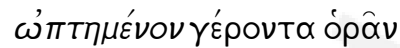

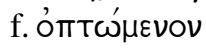

ruvaIkeía

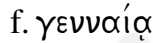

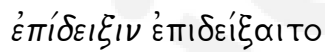

f. TíOTIV

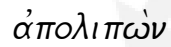

1. $\alpha$ пाо入 $\varepsilon^{i} \pi \omega \nu$

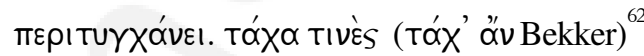

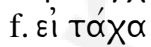

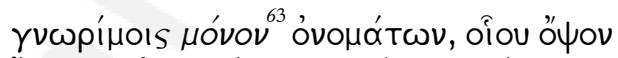

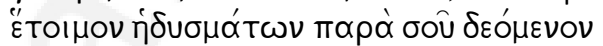

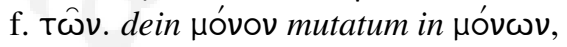

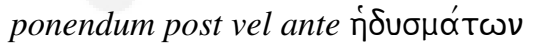

199. Dem. enc. 12 (III.267.7) $\quad \mu \eta \delta^{\prime}$ oûv

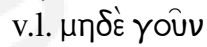

200. Dem.enc. 17 (III.269.18) $\quad \mu \eta \delta$ वं 1. $\mu$ กो

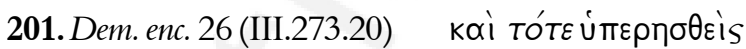
f. Toútw

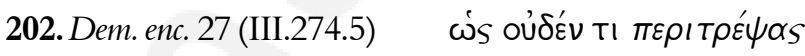
$\pi \propto \rho \alpha т \rho \dot{\psi} \psi \alpha \varsigma(E \Delta)$

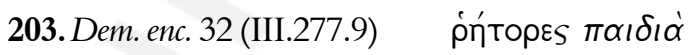
$\pi \alpha ı \delta^{\prime} \alpha(\mathrm{E} \Delta)$

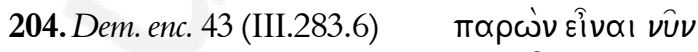
dele vิ̂v

\footnotetext{
${ }^{62}$ C'est ce que propose aussi Coray dans ses Observationes Miscellanea, p. 544: тóx'

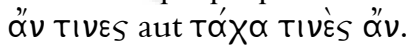

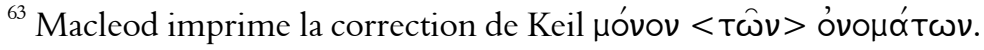




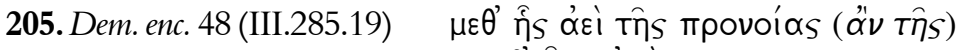

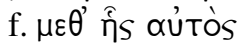

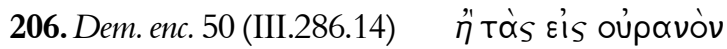

f. dele $\ddot{\eta}$

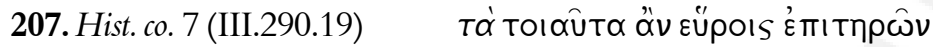

f. dele $\mathrm{T \alpha}$

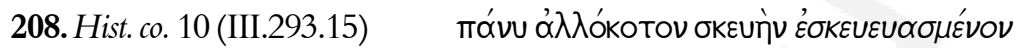

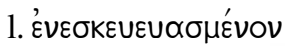

209. Hist. co. 17 (III.298.24)

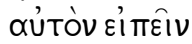

f. $\mu$ ’े

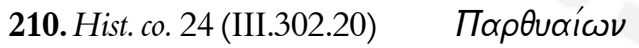

v.1. Пар日vâios

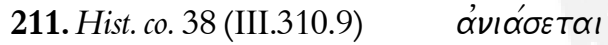

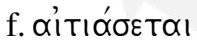

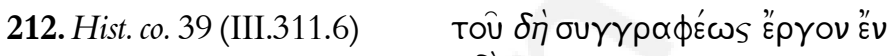

1. $\delta \dot{\varepsilon}$

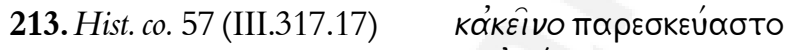

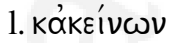

214. Sat. 1 (III.324.5)

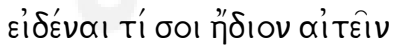

f. $\tau \varepsilon$ Tí

215. Sat. 4 (III.325.24)

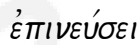

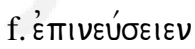

216. Sat. 6 (III.327.9-10)

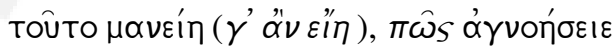

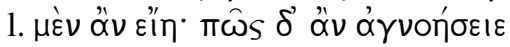

217. Sat. 16 (III.333.22)

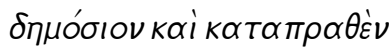

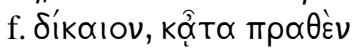

218. Sat. 19 (III.335.16)

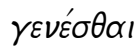

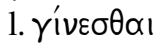

219. Sat. 22 (III.337.4)

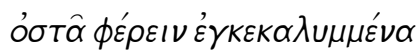
excidisse videtur vocabulum

220. Sat. 33 (III.342.19)

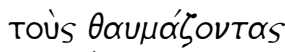

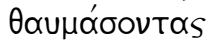

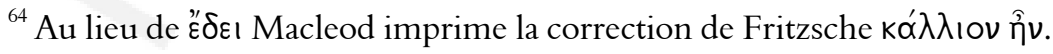

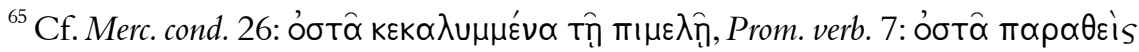

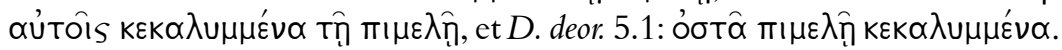


221. Sat. 36 (III.344.14)

222. Apol. 5 (III.369.14)

223. Apol. 8 (III.370.10-11)

224. Hes. 5 (III.381.14)

225. Hermot. 1 (IV.17.5)

226. Hermot. 4 (IV.19.13)

227. Hermot. 5 (IV.20.13)

228. Hermot. 7 (IV.23.3)

229. Hermot. 31 (IV.41.24)

230. Hermot. 37 (IV.46.21)

231. Hermot. 61 (IV.65.15)

232. Hermot. 73 (IV.75.21)

233. Hermot. 79 (IV.79.25)

234. Hermot. 79 (IV.79.28)

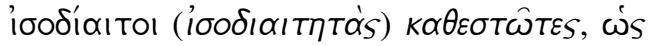

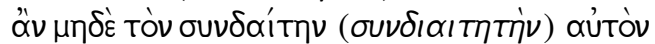

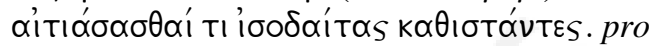

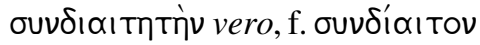

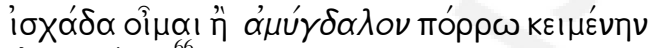
$\alpha_{\alpha u} \mu \delta \delta \alpha^{\prime} \lambda \eta v^{66}$

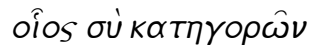

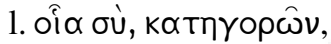

$\delta 1 \varepsilon \xi ı O ́ v T \varepsilon S$

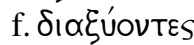

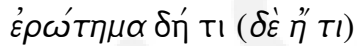

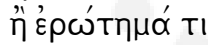

$\varepsilon \not k \alpha \zeta \varepsilon \nu$

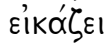

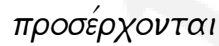

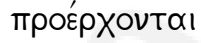

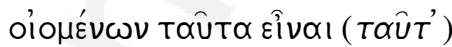

TI T\&ûT'

$\gamma \dot{\alpha} \rho \dot{\varepsilon} \pi 1 \pi \lambda \hat{\eta} \xi \alpha ।$

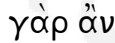

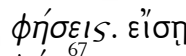

$\phi n$ 'n

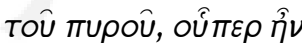

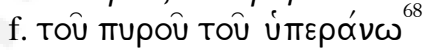

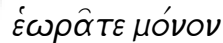

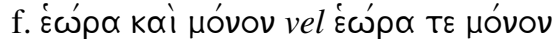

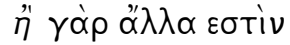

กิ

"ᄁ oûv oủxi

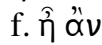

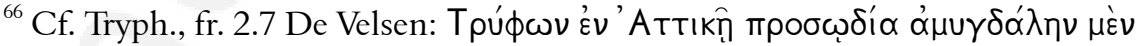

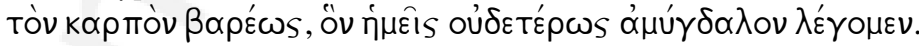

${ }^{67}$ Il s'agit d'une correction habituelle de la part de Coray: il pense que la dernière

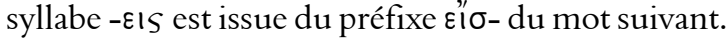

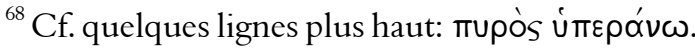


235. Hermot. 79 (IV.80.5)

236. Hermot. 84 (IV.83.5)

237. Prom. verb. 3 (IV.86.20)

238. Alc. 5 (IV.92.10)

239. Nav. 5 (IV.99.6)

240. Nav. 16 (IV.105.3-4)

241. Nav. 20 (IV.107.12)

242. Nav. 21 (IV.107.18)

243. Nav. 26 (IV.110.2)

244. Cyn. 4 (IV.138.2)

245. Cyn. 16 (IV.144.9)

246. Cyn. 18 (IV.145.10)

247.D. meretr. 8.2 (IV.338.9)

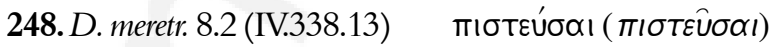

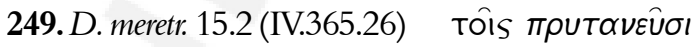

250. Philopatr. 6 (IV.372.6)

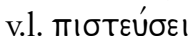

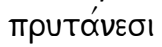

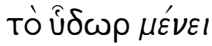

$\mu \varepsilon v \varepsilon \hat{\imath}$

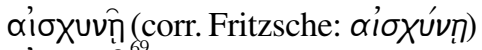

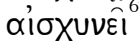

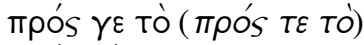

mpós tò $(E \Delta)$

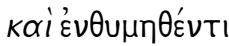

f. kờv

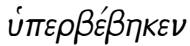

f. úTO $\beta^{\prime} \beta \eta K \varepsilon v$

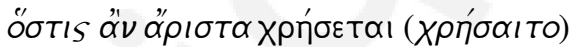

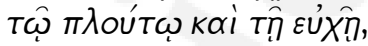

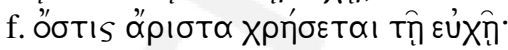

vid. var. lect.

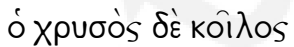

dele ó

$\mu \eta^{\prime} \mu \mathrm{O} \alpha^{\alpha} v \alpha^{\prime} \lambda \mathrm{v}$ ( $\mu \mathrm{ol}$ Fritzsche: $\mu$ ' codd.)

$\mu \mathrm{ou}$

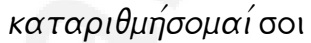

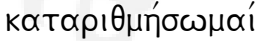

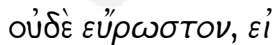

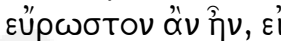

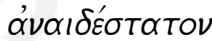

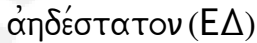

о̆точ

1. ӧто।

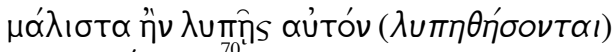

1. $\lambda \cup \pi \eta \eta \eta \dot{\sigma} \sigma \varepsilon \tau \alpha{ }^{70}$

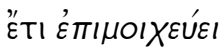

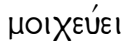

${ }^{69}$ Dans ses Observationes Miscellanex, p. 477, il devance Fritzsche: lege aíðuvị in futuro.

${ }^{70}$ Cf. aussi ses Observationes Miscellanea, p. 537: an $\lambda u \pi \eta \theta \dot{\sigma} \sigma \varepsilon \tau \propto เ ?$ 


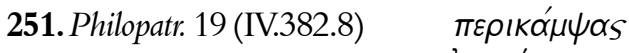

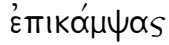

252. Ner. 2 (IV.406.11)

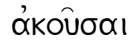

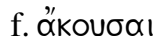

253. Ner. 3 (IV.406.13)

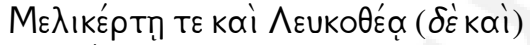
dele $\delta \dot{\varepsilon}$

254. Ner. 8 (IV.408.15)

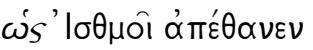
ôs

\section{Index des passages corrigés}

Adv. ind. 18: 154.

Adv. ind. 24: 156.

Alc. 5: 238.

Alex. 6: 118.

Alex. 10: 119.

Alex. 48: 39.

Amor. 6: 122.

Amor. 11: 48.

Amor. 15: 50.

Anach. 9: 173.

Apol. 8: 223.

Asin. 6: 114.

Asin. 10: 27.

Asin. 18: 28.

Asin. 28: 29, 30.

Asin.37: 115.

Asin. 48: 116.

Asin. 56: 179.

Bacch. 4: 133.

Bis acc. 27: 110.

Cal. 24: 3.

Cat. 11: 103, 144.

Cat. 22: 145.

Charid. 17: 97.

Cyn. 4: 244.

Cyn. 18: 246.

Dear.jud. 3: 20.

Dem. enc. 1: 197.

Dem. enc. 10: 63, 198.

Dem. enc. 16: 64.

Dem. enc. 24: 65.

Dem. enc. 27: 202.
Adv. ind. 20: 155.

Adv. ind. 25: 157.

Alex. 5: 117.

Alex. 9: 180.

Alex. 23: 38.

Amor. 1: 121.

Amor. 10: 123.

Amor. 14: 49.

Anach. 1: 172.

Apol. 5: 222.

Asin. 4: 174.

Asin . 7: 26.

Asin. 16: 175.

Asin. 27: 176, 177.

Asin. 30: 31.

Asin. 40: 32, 178.

Asin. 51: 33, 34.

Astr. 29: 188.

Bis acc. 1: 108, 109.

Cal. 23: 2.

Cat. 2: 143.

Cat. 19: 5.

Char. 1: 11.

Cyn. 1: 85.

Cyn. 16: 245.

D. deor. 2.4: 130.

Dear.jud. 7: 21.

Dem. enc. 5: 62.

Dem. enc. 12: 199.

Dem. enc. 17: 200.

Dem. enc. 26: 201.

Dem. enc. 32: 203. 
Dem.enc. 33: 66.

Dem. enc. 48: 205.

Demon. 10: 137.

D. meretr. 8.2: 247, 248.

D. meretr. 9.4: 89.

D. meretr. 15.2: 249.

D. mort. 20.6: 86.

Fug. 27: 57.

Gall. 8: 148.

Gall. 20: 149.

Herc. 4: 134.

Hermot. 4: 226.

Hermot. 7: 228.

Hermot. 21: 75.

Hermot. 32: 76.

Hermot. 52: 77.

Hermot. 61: 231.

Hermot. 74: 79.

Hermot. 84: 236.

Hes. 5: 224.

Hipp. 8: 132.

Hist. co. 7: 207.

Hist. co. 10: 208.

Hist. co. 24: 210.

Hist. co. 39: 212.

Hist. co. 57: 213.

Icar. 6: 8.

J. conf. 6: 146.

J.trag. 51: 147.

Jud. voc. 3: 141.

Lex. 7: 44.

Lex. 20: 46, 187.

Luct. 16: 35.

Macr. 1: 139.

Merc. cond. 5: 22.

Merc. cond. 10: 23.

Merc. cond. 26: 24, 25, 171.

Nav. 16: 240.

Nav. 21: 242.

Nav. 26: 243.

Nav. 34: 84.

Ner. 3: 253.

Nigr. 6: 135.

Nigr. 37: 99.

Paras. 1: 161.
Dem. enc. 43: 204.

Dem. enc. 50: 206.

D. meretr. 4.5: 87.

D. meretr. 9.3: 88.

D. meretr. 11.1: 90.

D. mort. 15.2: 128.

D. mort. 29.2: 129.

Fug. 33: 154.

Gall. 14: 7.

Gall. 24: 150.

Hermot. 1: 225.

Hermot. 5: 227.

Hermot. 17: 74.

Hermot. 31: 229.

Hermot. 37: 230.

Hermot. 55: 78.

Hermot. 73: 232.

Hermot. 79: 233, 234, 235.

Herod. 8: 72.

Hipp. 6: 98.

Hist. co. 3: 67.

Hist. co. 8: 68.

Hist. co. 17: 209.

Hist. co. 38: 211.

Hist. co. 51: 69, 70.

Icar. 3: 151.

Icar. 20: 9.

J. trag. 44: 6.

Jud. voc. 2: 4, 140.

Lex. 6: 43.

Lex. 12: 45.

Lex. 24: 47.

Luct. 20: 181.

Macr. 4: 101.

Merc. cond. 9: 170.

Merc. cond. 21: 113.

Nav. 5: 239.

Nav. 20: 81, 241.

Nav. 22: 82.

Nav. 32: 83.

Ner. 2: 252.

Ner. 8: 254.

Nigr. 34: 136.

Nigr. 38: 100.

Paras. 2: 14, 111. 
Paras. 3: 112.

Paras. 12: 15.

Paras. 42: 17.

Paras. 53: 164.

Peregr. 28: 191.

Phal.A 4: 131.

Philopatr. 3: 92.

Philopatr. 9:93.

Philopatr. 20:94.

Philopatr.22: 96.

Philops. 9: 167, 168.

Philops. 16: 19.

Pisc. 42: 153.

Pro im. 29: 189.

Prom. verb. 3: 80, 237.

Pseudol.16: 52, 124.

Pseudol.27: 54.

Rh. pr. 4:36.

Rh. pr. 11: 182.

Salt.26: 120.

Sat. 1: 214.

Sat. 4: 215.

Sat. 16: 217.

Sat. 22: 219.

Sat. 36: 221.

Somn. 3: 158.

Somn. 15: 160.

Syr. dea 30: 40.

Syr. dea 51: 41.

Tim. 6: 105.

Tim. 43: 10.

Tox. 12: 59.

Tox. 26: 60.

Tox. 46: 125.

Tox. 57: 126.

Tyr. 8: 55.

V.H.II 9: 102.

V.H.II 34: 139.

Vit. auct. 24: 12.
Paras. 8: 162.

Paras. 41: 16.

Paras. 51: 18, 163.

Paras. 57: 165.

Peregr. 37: 192, 193.

Philopatr. 1: 91.

Philopatr. 6: 250.

Philopatr. 19: 251.

Philopatr. 21: 95.

Philops. 8: 166.

Philops. 12: 169.

Pisc. 40: 13.

Pisc. 47: 107.

Prom. 5: 104.

Pseudol. 6: 51.

Pseudol. 20: 53.

Pseudol.30: 190.

Rh. pr. 10: 37.

Rh.pr. 23: 183.

Salt. 29: 186.

Sat. 2: 71.

Sat. 6: 216.

Sat. 19: 218.

Sat. 33: 220.

Sol. 7: 142.

Somn. 10: 159.

Syr. dea 11: 184.

Syr. dea 45: 185.

Syr. dea 60: 42.

Tim. 11: 106.

Tox. 10: 58.

Tox. 20: 195.

Tox. 34: 196.

Tox. 55: 61.

Tox. 61: 127.

Tyr. 10: 56.

V.H.II 30: 1.

Vit. auct. 14: 152.

Zeux. 9: 73. 


\section{Références}

ANDÒ V. Luciano: Il lutto. Palermo: Palumbo, 1984.

BAZOU, A. Adamantios Coray et ses corrections inédites sur Galien. In: BOUDON MILLOT, V.; GARZYA, A.; JOUANNA, J.; ROSELLI, A. (org.). Ecdotica e ricezione dei testi medici greci: Atti del V Convegno internazionale - Napoli, 12 ottobre 2004. Naples: M. D'Auria, 2006, p. 465-480.

BOMPAIRE, J. Lucien Euvres. Vols. I-III. Paris: Les Belles Lettres, 1993-2003.

CASEVITZ, M. La création verbale chez Lucien: le "Lexiphanes", Lexiphane et Lucien. In: BILLAULT, A. (org.). Lucien de Samosate: Actes du Colloque international de Lyon organisé au Centre d'études romaines et gallo-romaines, le 30 septembre - $1^{\text {er }}$ octobre 1993. Paris: De Boccard, 1994, p. 77-86.

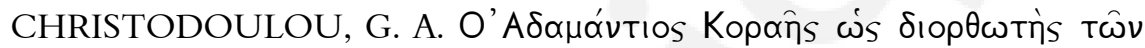

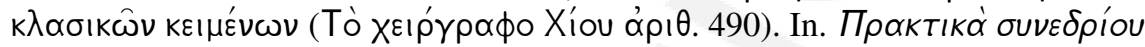
"Kopañs kai Xíos" (Xíos, 11-15 Maíiou 1983). Vol. I. Athènes: 'Ouńpeıv

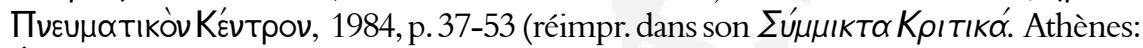
Édition privée, 1986, p. 237-255).

COENEN, J.Lukian Zeus Tragodos: Überlieferungsgeschichte, Text und Kommentar. Beiträge zur klassischen Philologie, 88. Meisenheim am Glan: Hain, 1977.

GARCÍA SOLER, M. J. El arte de comer en la antigua Grecia. Madrid: Bibliteca Nueva, 2001.

HENDERSON, J. The maculate muse: obscene language in Attic comedy. New York: Oxford University Press, 1991.

HOPKINSON, N. Lucian: a selection. Cambridge: Cambridge University Press, 2008.

JUFRESA, M.; MESTRE, F.; GÓMEZ, P. Luciano Obras III. Madrid: Consejo Superior de Investigaciones Científicas, 2000.

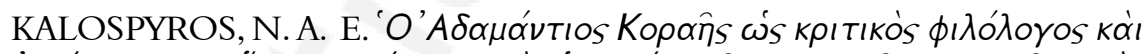

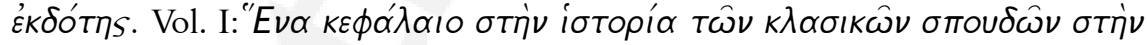

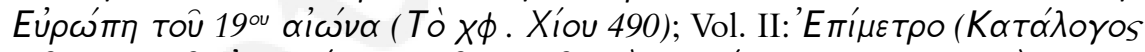

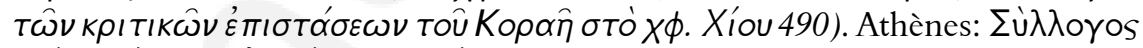

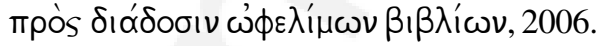

KAMBITSIS, J.L'Antiope d'Euripide. Athènes: E. Hourzamanis, 1972.

KARAVAS, O. Lucien et la tragédie. Untersuchungen zur antiken Literatur und Geschichte, 76. Berlin/ New York: W. de Gruyter, 2005.

KARAVAS, O. Luciano, los cristianos y Jesucristo. In: MESTRE, F.; GÓMEZ, P. (org.). Lucian of Samosata, Greek writer and Roman citizen. Barcelone: Publicacions i Edicions de la Universitat de Barcelona, 2010, p. 115-120.

LIGHTFOOT, J. L. Lucian: "On the Syrian goddess". Oxford: Oxford University Press, 2003. 
MACLEOD, M. D. Lucian. Vol. VIII. London/ New York: Harvard University Press, 1967.

MACLEOD, M. D. Luciani Opera. Vols. I-IV. Oxford: Oxford University Press, 1972-1987.

MESTRE, F.; GÓMEZ, P. Luciano Obras IV. Madrid: Consejo Superior de Investigaciones Científicas, 2007.

NESSELRATH, H. G., c.r. de MACLEOD, M. D. Luciani Opera. Vols. I-III. Oxford: Oxford University Press, 1972-1980 (Gnomon, Munich/Berlin, vol. 56, p. 577-609, 1984).

NESSELRATH, H. G. Lukians Parasitendialog: Untersuchungen und Kommentar. Untersuchungen zur antiken Literatur und Geschichte 22. Berlin/ New York: W. de Gruyter, 1985.

NESSELRATH, c.r. de MACLEOD, M. D. Luciani Opera. Vol. IV. Oxford: Oxford University Press, 1987 (Gnomon, Munich/ Berlin, vol. 62, p. 498-511, 1990).

RUSSO, G. Su una "crux" in Luciano, "Pseudologista" 16. Annali della Facoltà di lettere e filosofia dell'Università degli studi di Bari. Bari, vol. 42, p. 47-52, 1999.

VON MÖLLENDORFF, P. Lukian Hermotimos oder Lohnt es sich, Philosophie zu studieren? Texte zur Forschung, 74. Darmstadt: Gunter Narr, 2000.

WEISSENBERGER, M. Literaturtheorie bei Lukian: Untersuchungen zum Dialog "Lexiphanes". Beiträge zur Altertumskunde, 64. Stuttgart/ Leipzig: Teubner, 1996. ZWEIMÜLLER, S. Lukian "Rhetorum praeceptor": Einleitung, Text und Kommentar. Hypomnemata, 176. Göttingen: Vandenhoeck \& Ruprecht, 2008. 